\title{
Maximum principle for stochastic optimal control problem of forward-backward stochastic difference systems
}

\author{
Shaolin Ji* Haodong $\mathrm{Liu}^{\dagger}$
}

January 1, 2019

\begin{abstract}
In this paper, we study the maximum principle for stochastic optimal control problems of forward-backward stochastic difference systems (FBS $\Delta$ Ss). Two types of FBS $\Delta$ Ss are investigated. The first one is described by a partially coupled forward-backward stochastic difference equation (FBS $\Delta \mathrm{E}$ ) and the second one is described by a fully coupled FBS $\Delta \mathrm{E}$. By adopting an appropriate representation of the product rule and an appropriate formulation of the backward stochastic difference equation (BS $\Delta \mathrm{E})$, we deduce the adjoint difference equation. Finally, the maximum principle for this optimal control problem with the control domain being convex is established.
\end{abstract}

Keywords: backward stochastic difference equations; forward-backward stochastic difference equations; monotone condition; stochastic optimal control; maximum principle

\section{Introduction}

The Maximum Principle is one of the principal approaches in solving the optimal control problems. A lot of work has been done on the Maximum Principle for forward stochastic system. See, for example, Bensoussan [2], Bismut 4], Kushner [12], Peng [16]. Peng also firstly studied one kind of forward-backward stochastic control system (FBSCS) in [17] and obtained the maximum principle for this kind of control system with control domain being convex. The FBSCSs have wide applications in many fields. As the stochastic differential recursive utility, which is a generalization of a standard additive utility, can be regarded as a solution of a backward stochastic differential equation (BSDE). The recursive utility optimization problem can be described by a optimization problem for a FBSCS (see [19]). Besides, in the dynamic principalagent problem with unobservable states and actions, the principal's problem can be formulated as a partial information optimal control problem of a FBSCS (see [22]). We refer to [8], 11], [13], 21], 24], 25] for other works on optimization problems for FBSCSs.

In this paper, we will discuss the Maximum Principle for optimal control of discrete time systems described by forward-backward stochastic difference equations (FBS $\Delta$ Es). To the best of our knowledge, there are few

*Zhongtai Securities Institute for Financial Studies, Shandong University, Jinan, Shandong 250100, PR China. jsl@sdu.edu.cn. Research supported by NSF (No. 11571203).

${ }^{\dagger}$ Zhongtai Securities Institute for Financial Studies, Shandong University, Jinan, Shandong 250100, PR China. (Corresponding author). 
results on such optimization control problems. In fact, the discrete time control systems are of great value in practice. For example, the digital control can be formulated as discrete time control problems, where the sampled data is obtained at discrete instants of time. Besides, the forward-backward stochastic difference system (FBS $\Delta \mathrm{S}$ ) can be used for modeling in financial markets. For example, the solution to the backward stochastic difference equation (BS $\Delta \mathrm{E}$ ) can be used to construct time-consistent nonlinear expectations (see [5, [6]) and be used for pricing in the financial markets (see [3]). However, the formulation of BS $\Delta \mathrm{E}$ is quite different from its continuous time counterpart. Many works are devoted to the study of BS $\Delta$ Es (see, e.g. [3], [5], [6], 20]). Based on the driving process, there are mainly two types of formulations of BS $\Delta$ Es. One is driving by a finite state process which takes values from the basis vectors (as in [5]) and the other is driving by a martingale with independent increments (as in [3]). For the latter case, the solution of the BS $\Delta \mathrm{E}$ is a triple of processes which is due to the discrete time version of the Kunita-Watanabe decomposition. In this paper, we adopt the second type of formulation to investigate the optimization problems for FBS $\Delta$ Ss.

Let $\left(\Omega, \mathcal{F},\left\{\mathcal{F}_{t}\right\}_{0 \leq t \leq T}, P\right)$ be a probability space, and $W_{t}$ be a martingale process with independent increments. Define the difference operator $\Delta$ as $\Delta V_{t}=V_{t+1}-V_{t}$. Here we consider two types of controlled FBS $\Delta$ Ss.

Problem 1 (partially coupled system):

The controlled system is

$$
\left\{\begin{aligned}
\Delta X_{t} & =b\left(t, X_{t}, u_{t}\right)+\sum_{i=1}^{d} \sigma_{i}\left(t, X_{t}, u_{t}\right) \Delta W_{t}^{i}, \\
X_{0} & =x_{0} \\
\Delta Y_{t} & =-f\left(t+1, X_{t+1}, Y_{t+1}, Z_{t+1}, u_{t+1}\right)+Z_{t} \Delta W_{t}+\Delta N_{t}, \\
Y_{T} & =y_{T}
\end{aligned}\right.
$$

and the cost functional is

$$
J(u(\cdot))=\mathbb{E}\left[\sum_{t=0}^{T-1} l\left(t, X_{t}, Y_{t}, Z_{t}, u_{t}\right)+h\left(X_{T}\right)\right] .
$$

Problem 2 (fully coupled system):

The controlled system is:

$$
\left\{\begin{aligned}
\Delta X_{t} & =b\left(t, X_{t}, Y_{t}, Z_{t}, u_{t}\right)+\sum_{i=1}^{d} \sigma_{i}\left(t, X_{t}, Y_{t}, Z_{t}, u_{t}\right) \Delta W_{t}^{i}, \\
X_{0} & =x_{0}, \\
\Delta Y_{t} & =-f\left(t+1, X_{t+1}, Y_{t+1}, Z_{t+1}, u_{t+1}\right)+Z_{t} \Delta W_{t}+\Delta N_{t}, \\
Y_{T} & =y_{T},
\end{aligned}\right.
$$

and the cost functional is

$$
J(u(\cdot))=\mathbb{E}\left[\sum_{t=0}^{T-1} l\left(t, X_{t}, Y_{t}, Z_{t}, u_{t}\right)+h\left(X_{T}\right)\right] .
$$


Let $\left\{U_{t}\right\}_{t \in\{0,1, \ldots, T-1\}}$ be a sequence of nonempty convex subset of $\mathbb{R}^{r}$. We denote the set of admissible controls $\mathcal{U}$ by $\mathcal{U}=\left\{u(\cdot) \in \mathcal{M}^{2}\left(0, T-1 ; \mathbb{R}^{r}\right) \mid u(t) \in U_{t}\right\}$. It can be seen that in Problem $1, b$ and $\sigma$ do not contain the solution $(Y, Z)$ of the backward equation. This kind of FBS $\Delta \mathrm{E}$ is called the partially coupled FBS $\Delta$ E. Meanwhile, the system in Problem 2 is called the fully coupled FBS $\Delta$ E.

The optimal control problem is to find the optimal control $u \in \mathcal{U}$, such that the optimal control and the corresponding state trajectory can minimize the cost functional $J(u(\cdot))$. In this paper, we assume the control domain is convex. By making the perturbation of the optimal control at a fixed time point, we obtain the maximum principle for problem 1 and 2 .

To build the maximum principle, the key step is to find the adjoint variables which can be applied to deduce the variational inequality. In [14, the authors studied the maximum principle for a discrete time stochastic optimal control problem in which the state equation is only governed by a forward stochastic difference equation. By applying the Riesz representation theorem, they explicitly obtained the adjoint variables and establish the maximum principle. But to solve our problems, we need to construct the adjoint difference equations since generally the adjoint variables can not be obtained explicitly for our case. To construct the adjoint equations in our discrete time framework, the techniques which are adopted for the continuous time framework as in [16, 17] are not appliable. In this paper, we propose two techniques to deduce the adjoint difference equations. The first one is that we choose the following product rule:

$$
\Delta\left\langle X_{t}, Y_{t}\right\rangle=\left\langle X_{t+1}, \Delta Y_{t}\right\rangle+\left\langle\Delta X_{t}, Y_{t}\right\rangle
$$

where $X_{t}$ (resp. $Y_{t}$ ) subjects to a forward (resp. backward) stochastic difference equation. The second one is that the BS $\Delta \mathrm{E}$ should be formulated as in (2.1). In other words, the generator $f$ of the BS $\Delta \mathrm{E}$ (2.1) depends on time $t+1$. It is worth pointing out that this kind of formulation is just the formulation of the adjoint equations for stochastic optimal control problems (see 14 for the classical case). Based on these two techniques, we can deduce the adjoint difference equations. The readers may refer to Remark 3.6 for more details.

The remainder of this paper is organized as follows. In section 2 , two types of the controlled FBS $\Delta \mathrm{Ss}$ are formulated. We deduce the maximum principle for the partially coupled controlled FBS $\Delta \mathrm{S}$ in section 3 . Finally, we establish the maximum principle for the fully coupled controlled FBS $\Delta \mathrm{S}$ in section 4 .

\section{Preliminaries and model formulation}

Let $T$ be a deterministic terminal time, and let $\mathcal{T}:=\{0,1, \ldots, T\}$. Consider a filtered probability space $\left(\Omega, \mathcal{F},\left\{\mathcal{F}_{t}\right\}_{0 \leq t \leq T}, P\right)$, with $\mathcal{F}_{0}=\{\emptyset, \Omega\}$ and $\mathcal{F}=\mathcal{F}_{T}$. Here we define the difference operator $\Delta$ as $\Delta U_{t}=$ $U_{t+1}-U_{t}$. Let $W$ be a fixed $\mathbb{R}^{d}$-valued square integrable martingale process with independent increments, i.e. $\mathbb{E}\left[\Delta W_{t} \mid \mathcal{F}_{t}\right]=\mathbb{E}\left[\Delta W_{t}\right]=0$ for any $t \in\{0, \ldots, T-1\}$. Also we suppose that $\mathbb{E}\left[\Delta W_{t}\left(\Delta W_{t}\right)^{*}\right]=I_{d}$ for any $t \in\{0, \ldots, T-1\}$. Here $(\cdot)^{*}$ denotes vector transposition. We assume that $\mathcal{F}_{t}$ is the completion of the $\sigma$-algebra generated by the process $W$ up to time $t$.

Denote by $L^{2}\left(\mathcal{F}_{t} ; \mathbb{R}^{n}\right)$ the set of all $\mathcal{F}_{t}$-measurable square integrable random variable $X_{t}$ taking values in $\mathbb{R}^{n}$ and by $\mathcal{M}^{2}\left(0, t ; \mathbb{R}^{n}\right)$ the set of all $\left\{\mathcal{F}_{s}\right\}_{0 \leq s \leq t}$-adapted square integrable process $X$ taking values in $\mathbb{R}^{n}$. 
Moreover, we define $e_{i}=(0,0, \ldots, 0,1,0, \ldots, 0)^{*} \in \mathbb{R}^{n}$ and mention that an inequality on a vector quantity is to hold componentwise.

Consider the following backward stochastic difference equation (BS $\Delta \mathrm{E})$ :

$$
\left\{\begin{aligned}
\Delta Y_{t} & =-f\left(t+1, Y_{t+1}, Z_{t+1}\right)+Z_{t} \Delta W_{t}+\Delta N_{t}, \\
Y_{T} & =\eta,
\end{aligned}\right.
$$

where $\eta \in L^{2}\left(\mathcal{F}_{T} ; \mathbb{R}^{n}\right), f: \Omega \times\{1,2, \ldots, T\} \times \mathbb{R}^{n} \times \mathbb{R}^{n \times d} \longmapsto \mathbb{R}^{n}$.

Assumption 2.1 A1. The function $f(t, y, z)$ is uniformly Lipschitz continuous and independent of $z$ at $t=T$, i.e. there exists constants $c_{1}, c_{2}>0$, such that for any $t \in\{1,2, \ldots, T-1\}, y_{1}, y_{2} \in \mathbb{R}^{n}, z_{1}, z_{2} \in \mathbb{R}^{n \times d}$,

$$
\begin{aligned}
\left|f\left(T, y_{1}, z_{1}\right)-f\left(T, y_{2}, z_{2}\right)\right| & \leq c_{1}\left|y_{1}-y_{2}\right| \\
\left|f\left(t, y_{1}, z_{1}\right)-f\left(t, y_{2}, z_{2}\right)\right| & \leq c_{1}\left|y_{1}-y_{2}\right|+c_{2}\left\|z_{1}-z_{2}\right\|, \quad P-a . s .
\end{aligned}
$$

A2. $f(t, 0,0) \in L^{2}\left(\mathcal{F}_{t} ; \mathbb{R}^{n}\right)$ for any $t \in\{1,2, \ldots, T\}$.

Remark 2.2 The BSAE (2.1) is analogous to the continuous time BSDE driven by a general martingale (cf. [9]), and the solution is a triple of processes.

Definition 2.3 A solution to BSAE (2.1) is a triple of processes $(Y, Z, N) \in \mathcal{M}^{2}\left(0, T ; \mathbb{R}^{n}\right) \times \mathcal{M}^{2}\left(0, T-1 ; \mathbb{R}^{n \times d}\right) \times$ $\mathcal{M}^{2}\left(0, T ; \mathbb{R}^{n}\right)$ which satisfies equality (2.1) for all $t \in\{0,1, \ldots, T-1\}$, and $N$ is a martingale process strongly orthogonal to $W$.

By using the Galtchouk-Kunita-Watanabe decomposition in [3, we can obtain the existence and uniqueness result of $\mathrm{BS} \Delta \mathrm{E}$ (2.1):

Theorem 2.4 Suppose that Assumption (2.1) holds. Then for any terminal condition $\eta \in L^{2}\left(\mathcal{F}_{T} ; \mathbb{R}^{n}\right)$, the $B S \Delta E$ (2.1) has a unique adapted solution $(Y, Z, N)$.

Proof. We first prove the existence and uniqueness of $\left(Y_{T-1}, Z_{T-1}, \Delta N_{T-1}\right)$. Due to Assumption (2.1) and $\eta \in L^{2}\left(\mathcal{F}_{T} ; \mathbb{R}^{n}\right)$, we get $f(T, \eta) \in L^{2}\left(\mathcal{F}_{T} ; \mathbb{R}^{n}\right)$. Here we omit the variable $Z$ since $f$ is independent of $Z$ at time $T$. Then we have $\mathbb{E}\left[\left|\mathbb{E}\left[\eta+f(T, \eta) \mid \mathcal{F}_{T-1}\right]\right|^{2}\right]<\infty$. Hence, $\eta+f(T, \eta)-\mathbb{E}\left[\eta+f(T, \eta) \mid \mathcal{F}_{T-1}\right]$ is a square integrable martingale difference. So it admits the Galtchouk-Kunita-Watanabe decomposition, which implies that there exists $Z_{T-1} \in \mathcal{F}_{T-1}, Z_{T-1} \Delta W_{T-1} \in L^{2}\left(\mathcal{F}_{T} ; \mathbb{R}^{n}\right), \Delta N_{T-1} \in L^{2}\left(\mathcal{F}_{T} ; \mathbb{R}^{n}\right)$ such that $\mathbb{E}\left[\Delta N_{T-1} \mid \mathcal{F}_{T-1}\right]=0, \mathbb{E}\left[e_{i}^{*} \Delta N_{T-1}\left(\Delta W_{T-1}\right)^{*} \mid \mathcal{F}_{T-1}\right]=0$ and

$$
\eta+f(T, \eta)-\mathbb{E}\left[\eta+f(T, \eta) \mid \mathcal{F}_{T-1}\right]=Z_{T-1} \Delta W_{T-1}+\Delta N_{T-1} .
$$

Moreover, $\Delta N_{T-1}$ is uniquely determined in this decomposition. For fixed $i \in\{1,2, \ldots, n\}$, premultiply the equation by $e_{i}^{*}$, postmultiply the equation by $\left(\Delta W_{T-1}\right)^{*}$ and then take the $\mathcal{F}_{T-1}$ conditional expectation. This yields that

$$
\mathbb{E}\left[e_{i}^{*}(\eta+f(T, \eta))\left(\Delta W_{T-1}\right)^{*} \mid \mathcal{F}_{T-1}\right]=e_{i}^{*} Z_{T-1}
$$


since $\mathbb{E}\left[\Delta W_{T-1}\left(\Delta W_{T-1}\right)^{*} \mid \mathcal{F}_{T-1}\right]=I$. Therefore, we get the unique $Z_{T-1}$ by

$$
Z_{T-1}=\mathbb{E}\left[(\eta+f(T, \eta))\left(\Delta W_{T-1}\right)^{*} \mid \mathcal{F}_{T-1}\right]
$$

and

$$
\mathbb{E}\left[\left\|Z_{T-1}\right\|^{2}\right] \leq \mathbb{E}\left[\mathbb{E}\left[|\eta+f(T, \eta)|^{2} \mid \mathcal{F}_{T-1}\right] \mathbb{E}\left[\left|\left(\Delta W_{T-1}\right)\right|^{2} \mid \mathcal{F}_{T-1}\right]\right]<\infty .
$$

It leads that $Y_{T-1}=\mathbb{E}\left[\eta+f(T, \eta) \mid \mathcal{F}_{T-1}\right]$ and $Y_{T-1} \in L^{2}\left(\mathcal{F}_{T-1} ; \mathbb{R}^{n}\right)$.

Then, by similar arguments as above, we can obtain the unique solution $\left(Y_{t}, Z_{t}, \Delta N_{t}\right) \in L^{2}\left(\mathcal{F}_{t} ; \mathbb{R}^{n}\right) \times$ $L^{2}\left(\mathcal{F}_{t} ; \mathbb{R}^{n \times d}\right) \times L^{2}\left(\mathcal{F}_{t} ; \mathbb{R}^{n}\right)$ for $t \in\{0,1, \ldots, T-2\}$. Moreover,

$$
\begin{aligned}
Z_{t} & =\mathbb{E}\left[\left(Y_{t+1}+f\left(t+1, Y_{t+1}, Z_{t+1}\right)\right)\left(\Delta W_{t}\right)^{*} \mid \mathcal{F}_{t}\right], \\
Y_{t} & =\mathbb{E}\left[Y_{t+1}+f\left(t+1, Y_{t+1}, Z_{t+1}\right) \mid \mathcal{F}_{t}\right] .
\end{aligned}
$$

By taking the convention $N_{0}=0$ and letting $N_{t}=N_{0}+\sum_{s=0}^{t-1} \Delta N_{s}$, we have that (2.1) holds true for all $t \in\{0,1, \ldots, T-1\}$. Finally, since

$$
\begin{aligned}
& \mathbb{E}\left[e_{i}^{*} N_{t}\left(W_{t}\right)^{*} \mid \mathcal{F}_{t-1}\right] \\
& =e_{i}^{*} \sum_{s=0}^{t-2} \Delta N_{s} \mathbb{E}\left[\left(W_{t}\right)^{*} \mid \mathcal{F}_{t-1}\right]+\mathbb{E}\left[e_{i}^{*} \Delta N_{t-1}\left(W_{t-1}+\Delta W_{t-1}\right)^{*} \mid \mathcal{F}_{t-1}\right] \\
& =e_{i}^{*} N_{t-1}\left(W_{t-1}\right)^{*}
\end{aligned}
$$

we conclude that $N$ is strongly orthogonal to $W$.

Now we consider the control systems (1.1)-(1.2) and (1.3)-(1.4).

Let the coefficients in system (1.1)-(1.2) be such that:

$$
\begin{aligned}
b(\omega, t, x, u) & : \Omega \times\{0,1, \ldots, T-1\} \times \mathbb{R}^{m} \times \mathbb{R}^{r} \rightarrow \mathbb{R}^{m}, \\
\sigma_{i}(\omega, t, x, u) & : \Omega \times\{0,1, \ldots, T-1\} \times \mathbb{R}^{m} \times \mathbb{R}^{r} \rightarrow \mathbb{R}^{m}, \\
f(\omega, t, x, y, z, u) & : \Omega \times\{1,2, \ldots, T\} \times \mathbb{R}^{m} \times \mathbb{R}^{n} \times \mathbb{R}^{n \times d} \times \mathbb{R}^{r} \rightarrow \mathbb{R}^{n}, \\
l(\omega, t, x, y, z, u) & : \Omega \times\{0,1, \ldots, T-1\} \times \mathbb{R}^{m} \times \mathbb{R}^{n} \times \mathbb{R}^{n \times d} \times \mathbb{R}^{r} \rightarrow \mathbb{R}, \\
h(\omega, x) & : \Omega \times \mathbb{R}^{m} \rightarrow \mathbb{R} .
\end{aligned}
$$

And the coefficients in system (1.3)-(1.4) be such that:

$$
\begin{aligned}
b(\omega, t, x, y, z, u) & : \Omega \times\{0,1, \ldots, T-1\} \times \mathbb{R}^{n} \times \mathbb{R}^{n} \times \mathbb{R}^{n \times d} \times \mathbb{R}^{r} \rightarrow \mathbb{R}^{n}, \\
\sigma_{i}(\omega, t, x, y, z, u) & : \Omega \times\{0,1, \ldots, T-1\} \times \mathbb{R}^{n} \times \mathbb{R}^{n} \times \mathbb{R}^{n \times d} \times \mathbb{R}^{r} \rightarrow \mathbb{R}^{n}, \\
f(\omega, t, x, y, z, u) & : \Omega \times\{1,2, \ldots, T\} \times \mathbb{R}^{n} \times \mathbb{R}^{n} \times \mathbb{R}^{n \times d} \times \mathbb{R}^{r} \rightarrow \mathbb{R}^{n}, \\
l(\omega, t, x, y, z, u) & : \Omega \times\{0,1, \ldots, T-1\} \times \mathbb{R}^{n} \times \mathbb{R}^{n} \times \mathbb{R}^{n \times d} \times \mathbb{R}^{r} \rightarrow \mathbb{R}, \\
h(\omega, x) & : \Omega \times \mathbb{R}^{n} \rightarrow \mathbb{R} .
\end{aligned}
$$

Remark 2.5 The cost functional in [17] consists of three parts: the running cost functional, the terminal cost functional of $X_{T}$, the initial cost functional of $Y_{0}$. In our formulation, if we take $l\left(\omega, 0, X_{0}, Y_{0}, Z_{0}, u_{0}\right)=$ $\gamma\left(\omega, Y_{0}\right)$, then the cost functional 1.4) for our discrete time framework can be reduced to the cost functional in [17] formally. 
For system (1.1)-(1.2), we assume that:

Assumption 2.6 For $\varphi=b, \sigma_{i}, f, l, h$, we assume that

1. $\varphi$ is adapted map, i.e. for any $(x, y, z, u) \in \mathbb{R}^{m} \times \mathbb{R}^{n} \times \mathbb{R}^{n \times d} \times \mathbb{R}^{r}, \varphi(\cdot, \cdot, x, y, z, u)$ is $\left\{\mathcal{F}_{t}\right\}$-adapted process. Moreover, $\varphi(\cdot, t, 0,0,0,0) \in L^{2}\left(\mathcal{F}_{t}\right)$.

2. $\forall t \in\{0,1, \ldots, T\}, \varphi(\cdot, t, \cdot, \cdot, \cdot, \cdot)$ is continuously differentiable with respect to $x, y, z, u$, and $\varphi_{x}, \varphi_{y}, \varphi_{z_{i}}, \varphi_{u}$ are uniformly bounded $P-$ a.s.. Also, for $t=T, f_{z_{i}} \equiv 0$, i.e. $f$ is independent of $z$ at time T. Here we use $z_{i}$ to represent the $i$-th column of the matrix $z$.

Let

$$
\lambda=\left(\begin{array}{c}
x \\
y \\
z
\end{array}\right), A(t, \lambda ; u)=\left(\begin{array}{c}
-f \\
b \\
\sigma
\end{array}\right)(t, \lambda ; u) .
$$

For control system (1.3)-(1.4), we additionally assume that:

Assumption 2.7 For any $u \in \mathcal{U}$, the coefficients in (1.3) satisfy the following monotone conditions, i.e. when $t \in\{1, \ldots, T-1\}$,

$$
\begin{gathered}
\left\langle A\left(t, \lambda_{1} ; u\right)-A\left(t, \lambda_{2} ; u\right), \lambda_{1}-\lambda_{2}\right\rangle \leq-\alpha\left|\lambda_{1}-\lambda_{2}\right|^{2}, P-\text { a.s. } \\
\forall \lambda_{1}, \lambda_{2} \in \mathbb{R}^{n} \times \mathbb{R}^{n} \times \mathbb{R}^{n} ;
\end{gathered}
$$

when $t=T$,

$$
\left\langle-f\left(T, x_{1}, y, z, u\right)+f\left(T, x_{2}, y, z, u\right), x_{1}-x_{2}\right\rangle \leq-\alpha\left|x_{1}-x_{2}\right|^{2}, P-a . s .
$$

when $t=0$,

$$
\begin{aligned}
& \left\langle b\left(0, \lambda_{1} ; u\right)-b\left(0, \lambda_{2} ; u\right), y_{1}-y_{2}\right\rangle+\left\langle\sigma\left(0, \lambda_{1} ; u\right)-\sigma\left(0, \lambda_{2} ; u\right), z_{1}-z_{2}\right\rangle \\
& \leq-\alpha\left[\left|y_{1}-y_{2}\right|^{2}+\left\|z_{1}-z_{2}\right\|^{2}\right]
\end{aligned}
$$

where $\alpha$ is a given positive constant.

Besides, in the following, we formally denote $b(T, x, y, z, u) \equiv 0, \sigma(T, x, y, z, u) \equiv 0, l(T, x, y, z, u) \equiv 0$, $f(0, x, y, z, u) \equiv 0$.

\section{Maximum principle for the partially coupled FBS $\Delta \mathrm{E}$ system}

For any $u \in \mathcal{U}$, it is obvious that there exists a unique solution $\left\{X_{t}\right\}_{t=0}^{T} \in \mathcal{M}^{2}\left(0, T ; \mathbb{R}^{m}\right)$ to the forward stochastic difference equation in the system (1.1). Then, by Theorem 2.4, the backward equation in the system (1.1) has a unique solution $(Y, Z, N)$ where $Y=\left\{Y_{t}\right\}_{t=0}^{T}, Z=\left\{Z_{t}\right\}_{t=0}^{T-1}$ and $N=\left\{N_{t}\right\}_{t=0}^{T}$. 
Suppose that $\bar{u}=\left\{\bar{u}_{t}\right\}_{t=0}^{T}$ is the optimal control of problem (1.1)-(1.2) and $(\bar{X}, \bar{Y}, \bar{Z})$ is the corresponding optimal trajectory. For a fixed time $0 \leq s \leq T$, choose any $\Delta v \in L^{2}\left(\mathcal{F}_{s} ; \mathbb{R}^{r}\right)$ such that $\bar{u}_{s}+\Delta v$ takes values in $U_{s}$. For any $\varepsilon \in[0,1]$, construct the perturbed admissible control

$$
u_{t}^{\varepsilon}=\left(1-\delta_{t s}\right) \bar{u}_{t}+\delta_{t s}\left(\bar{u}_{s}+\varepsilon \Delta v\right)=\bar{u}_{t}+\delta_{t s} \varepsilon \Delta v,
$$

where $\delta_{t s}=1$ for $t=s, \delta_{t s}=0$ for $t \neq s$ and $t \in\{0,1, \ldots, T\}$. Since $U_{s}$ is a convex set, $\left\{u_{t}^{\varepsilon}\right\}_{t=0}^{T} \in \mathcal{U}$ is an admissible control. Let $\left(X^{\varepsilon}, Y^{\varepsilon}, Z^{\varepsilon}, N^{\varepsilon}\right)$ be the solution of (1.1) corresponding to the control $u^{\varepsilon}$.

Set

$$
\begin{aligned}
\bar{\varphi}(t) & =\varphi\left(t, \bar{X}_{t}, \bar{Y}_{t}, \bar{Z}_{t}, \bar{u}_{t}\right), \quad \varphi^{\varepsilon}(t)=\varphi\left(t, X_{t}^{\varepsilon}, Y_{t}^{\varepsilon}, Z_{t}^{\varepsilon}, u_{t}^{\varepsilon}\right), \\
\widetilde{\varphi}^{\varepsilon}(t) & =\varphi\left(t, \bar{X}_{t}, \bar{Y}_{t}, \bar{Z}_{t}, u_{t}^{\varepsilon}\right), \quad \varphi_{\mu}(t)=\varphi_{\mu}\left(t, \bar{X}_{t}, \bar{Y}_{t}, \bar{Z}_{t}, \bar{u}_{t}\right),
\end{aligned}
$$

where $\varphi=b, \sigma_{i}, g, f, l, h$ and $\mu=x, y, z_{i}$ and $u$.

Then, we have the following estimates.

Lemma 3.1 Under Assumption (2.6), we have

$$
\sup _{0 \leq t \leq T} \mathbb{E}\left|X_{t}^{\varepsilon}-\bar{X}_{t}\right|^{2} \leq C \varepsilon^{2} \mathbb{E}|\Delta v|^{2}
$$

Proof. In the following, the positive constant $C$ may change from lines to lines.

When $t=0, \ldots, s, X_{t}^{\varepsilon}=\bar{X}_{t}$.

When $t=s+1$,

$$
X_{s+1}^{\varepsilon}-\bar{X}_{s+1}=\widetilde{b}^{\varepsilon}(s)-\bar{b}(s)+\sum_{i=1}^{d}\left[\widetilde{\sigma}_{i}^{\varepsilon}(s)-\bar{\sigma}_{i}(s)\right] \Delta W_{s}^{i}
$$

Then,

$$
\mathbb{E}\left|X_{s+1}^{\varepsilon}-\bar{X}_{s+1}\right|^{2} \leq(d+1) \mathbb{E}\left[\left|\widetilde{b}^{\varepsilon}(s)-\bar{b}(s)\right|^{2}+\sum_{i=1}^{d}\left|\left[\widetilde{\sigma}_{i}^{\varepsilon}(s)-\bar{\sigma}_{i}(s)\right] \Delta W_{s}^{i}\right|^{2}\right] .
$$

By the boundedness of $b_{u}$, we have

$$
\mathbb{E}\left[\left|\widetilde{b}^{\varepsilon}(s)-\bar{b}(s)\right|^{2}\right] \leq C \mathbb{E}\left[\left|u_{s}^{\varepsilon}-\bar{u}_{s}\right|^{2}\right]=C \varepsilon^{2} \mathbb{E}\left[|\Delta v|^{2}\right] .
$$

By the boundedness of $\sigma_{i u}$, we have

$$
\begin{aligned}
& \mathbb{E}\left|\left[\widetilde{\sigma}_{i}^{\varepsilon}(s)-\bar{\sigma}_{i}(s)\right] \Delta W_{s}^{i}\right|^{2} \\
& =\mathbb{E}\left[\left|\widetilde{\sigma}_{i}^{\varepsilon}(s)-\bar{\sigma}_{i}(s)\right|^{2} \mid \mathbb{E}\left[\left|\Delta W_{s}^{i}\right|^{2} \mid \mathcal{F}_{s}\right]\right] \\
& =\mathbb{E}\left[\left|\left[\widetilde{\sigma}_{i}^{\varepsilon}(s)-\bar{\sigma}_{i}(s)\right]\right|^{2}\right] \\
& \leq C \varepsilon^{2} \mathbb{E}\left[|\Delta v|^{2}\right]
\end{aligned}
$$

which leads to

$$
\mathbb{E}\left|X_{s+1}^{\varepsilon}-\bar{X}_{s+1}\right|^{2} \leq C \varepsilon^{2} \mathbb{E}\left[|\Delta v|^{2}\right]
$$


When $t=s+2, \ldots, T$,

$$
\begin{aligned}
\mathbb{E}\left|X_{t}^{\varepsilon}-\bar{X}_{t}\right|^{2} & \leq(d+1) \mathbb{E}\left|b\left(t-1, X_{t-1}^{\varepsilon}, \bar{u}_{t-1}\right)-b\left(t-1, \bar{X}_{t-1}, \bar{u}_{t-1}\right)\right|^{2} \\
& +\sum_{i=1}^{d}\left|\left[\sigma_{i}\left(t-1, X_{t-1}^{\varepsilon}, \bar{u}_{t-1}\right)-\sigma_{i}\left(t-1, \bar{X}_{t-1}, \bar{u}_{t-1}\right)\right] \Delta W_{s}^{i}\right|^{2}
\end{aligned}
$$

Due to the boundedness of $b_{x}, \sigma_{i x}$, we obtain $\mathbb{E}\left|X_{t}^{\varepsilon}-\bar{X}_{t}\right|^{2} \leq C \mathbb{E}\left[\left|X_{t-1}^{\varepsilon}-\bar{X}_{t-1}\right|^{2}\right]$. Thus, by induction we prove the result.

Let $\xi=\left\{\xi_{t}\right\}_{t=0}^{T}$ be the solution to the following difference equation,

$$
\left\{\begin{aligned}
\Delta \xi_{t} & =b_{x}(t) \xi_{t}+\delta_{t s} b_{u}(t) \varepsilon \Delta v+\sum_{i=1}^{d}\left[\sigma_{i x}(t) \xi_{t}+\delta_{t s} \varepsilon \sigma_{i u}(t) \Delta v\right] \Delta W_{t}^{i}, \\
\xi_{0} & =0
\end{aligned}\right.
$$

It is easy to check that

$$
\sup _{0 \leq t \leq T} \mathbb{E}\left|\xi_{t}\right|^{2} \leq C \varepsilon^{2} \mathbb{E}|\Delta v|^{2}
$$

and we have the following result:

Lemma 3.2 Under Assumption [2.6, we have

$$
\sup _{0 \leq t \leq T} \mathbb{E}\left|X_{t}^{\varepsilon}-\bar{X}_{t}-\xi_{t}\right|^{2}=o\left(\varepsilon^{2}\right) .
$$

Proof. When $t=0, \ldots, s, X_{t}^{\varepsilon}=\bar{X}_{t}$ and $\xi_{t}=0$ which lead to $X_{t}^{\varepsilon}-\bar{X}_{t}-\xi_{t}=0$.

When $t=s+1$,

$$
X_{s+1}^{\varepsilon}-\bar{X}_{s+1}-\xi_{s+1}=\left[\widetilde{b}_{u}(s)-b_{u}(s)\right] \varepsilon \Delta v+\sum_{i=1}^{d}\left[\widetilde{\sigma}_{i u}(s)-\sigma_{i u}(s)\right] \varepsilon \Delta v \Delta W_{s}^{i}
$$

where

$$
\begin{gathered}
\widetilde{b}_{u}(s)=\int_{0}^{1} b_{u}\left(s, \bar{X}_{s}, \bar{u}_{s}+\lambda\left(u_{s}^{\varepsilon}-\bar{u}_{s}\right)\right) d \lambda, \\
\widetilde{\sigma}_{i u}(s)=\int_{0}^{1} \sigma_{i u}\left(s, \bar{X}_{s}, \bar{u}_{s}+\lambda\left(u_{s}^{\varepsilon}-\bar{u}_{s}\right)\right) d \lambda .
\end{gathered}
$$

Then

$$
\begin{aligned}
\mathbb{E}\left|X_{s+1}^{\varepsilon}-\bar{X}_{s+1}-\xi_{s+1}\right|^{2} & \left.\leq\left.(d+1) \mathbb{E}\left[|| \widetilde{b}_{u}(s)-b_{u}(s)\right] \varepsilon \Delta v\right|^{2}+\sum_{i=1}^{d}\left|\left[\widetilde{\sigma}_{i u}(s)-\sigma_{i u}(s)\right] \varepsilon \Delta v \Delta W_{s}^{i}\right|^{2}\right] \\
& \leq C \mathbb{E}\left[\left\|\widetilde{b}_{u}(s)-b_{u}(s)\right\|^{2}|\Delta v|^{2}+\sum_{i=1}^{d}\left\|\widetilde{\sigma}_{i u}(s)-\sigma_{i u}(s)\right\|^{2}|\Delta v|^{2}\right] \varepsilon^{2} .
\end{aligned}
$$

Since $\left\|\widetilde{b}_{u}(s)-b_{u}(s)\right\| \rightarrow 0$ and $\left\|\widetilde{\sigma}_{i u}(s)-\sigma_{i u}(s)\right\| \rightarrow 0$ as $\varepsilon \rightarrow 0$, we have

$$
\lim _{\varepsilon \rightarrow 0} \frac{1}{\varepsilon^{2}} \mathbb{E}\left|X_{s+1}^{\varepsilon}-\bar{X}_{s+1}-\xi_{s+1}\right|^{2}=0 .
$$


When $t=s+2, \ldots, T$,

$$
\begin{aligned}
X_{t}^{\varepsilon}-\bar{X}_{t}-\xi_{t} & =\widetilde{b}_{x}(t-1)\left(X_{t-1}^{\varepsilon}-\bar{X}_{t-1}-\xi_{t-1}\right)+\left[\widetilde{b}_{x}(t-1)-b_{x}(t-1)\right] \xi_{t-1} \\
& +\sum_{i=1}^{d}\left\{\widetilde{\sigma}_{i x}(t-1)\left(X_{t-1}^{\varepsilon}-\bar{X}_{t-1}-\xi_{t-1}\right)+\left[\widetilde{\sigma}_{i x}(t-1)-\sigma_{i x}(t-1)\right] \xi_{t-1}\right\} \Delta W_{t-1}^{i}
\end{aligned}
$$

where

$$
\begin{gathered}
\widetilde{b}_{x}(t)=\int_{0}^{1} b_{x}\left(t, \bar{X}_{t}+\lambda\left(X_{t}^{\varepsilon}-\bar{X}_{t}\right), \bar{u}_{t}\right) d \lambda, \\
\widetilde{\sigma}_{i x}(t)=\int_{0}^{1} \sigma_{i x}\left(t, \bar{X}_{t}+\lambda\left(X_{t}^{\varepsilon}-\bar{X}_{t}\right), \bar{u}_{t}\right) d \lambda .
\end{gathered}
$$

Then

$$
\begin{aligned}
\mathbb{E}\left|X_{t}^{\varepsilon}-\bar{X}_{t}-\xi_{t}\right|^{2} \leq & C \mathbb{E}\left\|\widetilde{b}_{x}(t-1)\right\|^{2}\left|X_{t-1}^{\varepsilon}-\bar{X}_{t-1}-\xi_{t-1}\right|^{2}+\left\|\widetilde{b}_{x}(t-1)-b_{x}(t-1)\right\|^{2}\left|\xi_{t-1}\right|^{2} \\
& +\sum_{i=1}^{d}\left\|\widetilde{\sigma}_{i x}(t-1)\right\|^{2}\left|X_{t-1}^{\varepsilon}-\bar{X}_{t-1}-\xi_{t-1}\right|^{2}+\sum_{i=1}^{d}\left\|\widetilde{\sigma}_{i x}(t-1)-\sigma_{i x}(t-1)\right\|^{2}\left|\xi_{t-1}\right|^{2} \\
\leq & C \mathbb{E}\left(\left\|\widetilde{b}_{x}(t-1)\right\|^{2}+\sum_{i=1}^{d}\left\|\widetilde{\sigma}_{i x}(t-1)\right\|^{2}\right)\left|X_{t-1}^{\varepsilon}-\bar{X}_{t-1}-\xi_{t-1}\right|^{2} \\
& \left.+\left\|\widetilde{b}_{x}(t-1)-b_{x}(t-1)\right\|^{2}\left|\xi_{t-1}\right|^{2}+\sum_{i=1}^{d}\left\|\widetilde{\sigma}_{i x}(t-1)-\sigma_{i x}(t-1)\right\|^{2}\left|\xi_{t-1}\right|^{2}\right] .
\end{aligned}
$$

$\left\|\widetilde{b}_{x}(t-1)-b_{x}(t-1)\right\| \rightarrow 0$ and $\left\|\widetilde{\sigma}_{i x}(t-1)-\sigma_{i x}(t-1)\right\| \rightarrow 0$ as $\varepsilon \rightarrow 0$. Since $\widetilde{b}_{x}(t-1)$ and $\widetilde{\sigma}_{i x}(t-1)$ are bounded, by the estimation (3.5), we have

$$
\lim _{\varepsilon \rightarrow 0} \frac{1}{\varepsilon^{2}} \mathbb{E}\left|X_{t}^{\varepsilon}-\bar{X}_{t}-\xi_{t}\right|^{2}=0 .
$$

This completes the proof.

Lemma 3.3 Under Assumption 2.6, we have

$$
\begin{aligned}
\sup _{0 \leq t \leq T} \mathbb{E}\left|Y_{t}^{\varepsilon}-\bar{Y}_{t}\right|^{2} & \leq C \varepsilon^{2} \mathbb{E}|\Delta v|^{2} \\
\sup _{0 \leq t \leq T-1} \mathbb{E}\left\|Z_{t}^{\varepsilon}-\bar{Z}_{t}\right\|^{2} & \leq C \varepsilon^{2} \mathbb{E}|\Delta v|^{2} .
\end{aligned}
$$

Proof. It is obvious that $Y_{T}^{\varepsilon}-\bar{Y}_{T}=0$ at time $T$.

When $t=s, \ldots, T-1$ (if $s=T$, skip this part), we have

$$
\begin{aligned}
& \mathbb{E}\left|f\left(t+1, X_{t+1}^{\varepsilon}, Y_{t+1}^{\varepsilon}, Z_{t+1}^{\varepsilon}, \bar{u}_{t+1}\right)-f\left(t+1, \bar{X}_{t+1}, \bar{Y}_{t+1}, \bar{Z}_{t+1}, \bar{u}_{t+1}\right)\right|^{2} \\
& \leq C \mathbb{E}\left[\left|X_{t+1}^{\varepsilon}-\bar{X}_{t+1}\right|^{2}+\left|Y_{t+1}^{\varepsilon}-\bar{Y}_{t+1}\right|^{2}+\left\|Z_{t+1}^{\varepsilon}-\bar{Z}_{t+1}\right\|^{2}\right] \\
& \leq C \mathbb{E}\left[\left|Y_{t+1}^{\varepsilon}-\bar{Y}_{t+1}\right|^{2}+\left\|Z_{t+1}^{\varepsilon}-\bar{Z}_{t+1}\right\|^{2}\right]+C_{1} \varepsilon^{2} \mathbb{E}|\Delta v|^{2} .
\end{aligned}
$$

It yields that

$$
\mathbb{E}\left|Y_{t}^{\varepsilon}-\bar{Y}_{t}\right|^{2} \leq C \mathbb{E}\left[\left|Y_{t+1}^{\varepsilon}-\bar{Y}_{t+1}\right|^{2}+\left\|Z_{t+1}^{\varepsilon}-\bar{Z}_{t+1}\right\|^{2}\right]+C \varepsilon^{2} \mathbb{E}|\Delta v|^{2}
$$


Similarly, we have

$$
\begin{aligned}
& \mathbb{E}\left\|Z_{t}^{\varepsilon}-\bar{Z}_{t}\right\|^{2} \\
& \leq C \mathbb{E}\left[\left|Y_{t+1}^{\varepsilon}-\bar{Y}_{t+1}\right|^{2}+\left\|Z_{t+1}^{\varepsilon}-\bar{Z}_{t+1}\right\|^{2}\right]+C \varepsilon^{2} \mathbb{E}|\Delta v|^{2} .
\end{aligned}
$$

When $t=s-1$, by similar analysis,

$$
\left\{\begin{aligned}
\mathbb{E}\left|Y_{t}^{\varepsilon}-\bar{Y}_{t}\right|^{2} & \leq C \mathbb{E}\left[\left|Y_{t+1}^{\varepsilon}-\bar{Y}_{t+1}\right|^{2}+\left\|Z_{t+1}^{\varepsilon}-\bar{Z}_{t+1}\right\|^{2}\right]+C \varepsilon^{2} \mathbb{E}|\Delta v|^{2} \\
\mathbb{E}\left[\left\|Z_{t}^{\varepsilon}-\bar{Z}_{t}\right\|^{2}\right] & \leq C \mathbb{E}\left[\left|Y_{t+1}^{\varepsilon}-\bar{Y}_{t+1}\right|^{2}+\left\|Z_{t+1}^{\varepsilon}-\bar{Z}_{t+1}\right\|^{2}\right]+C \varepsilon^{2} \mathbb{E}|\Delta v|^{2}
\end{aligned}\right.
$$

If $s=T$, it shows like

$$
\left\{\begin{aligned}
\mathbb{E}\left|Y_{T-1}^{\varepsilon}-\bar{Y}_{T-1}\right|^{2} & \leq C \varepsilon^{2} \mathbb{E}|\Delta v|^{2} \\
\mathbb{E}\left[\left\|Z_{T-1}^{\varepsilon}-\bar{Z}_{T-1}\right\|^{2}\right] & \leq C \varepsilon^{2} \mathbb{E}|\Delta v|^{2}
\end{aligned}\right.
$$

When $t=0, \ldots, s-2$, we have

$$
\left\{\begin{aligned}
\mathbb{E}\left|Y_{t}^{\varepsilon}-\bar{Y}_{t}\right|^{2} & \leq C \mathbb{E}\left[\left|Y_{t+1}^{\varepsilon}-\bar{Y}_{t+1}\right|^{2}+\left\|Z_{t+1}^{\varepsilon}-\bar{Z}_{t+1}\right\|^{2}\right], \\
\mathbb{E}\left[\left\|Z_{t}^{\varepsilon}-\bar{Z}_{t}\right\|^{2}\right] & \leq C \mathbb{E}\left[\left|Y_{t+1}^{\varepsilon}-\bar{Y}_{t+1}\right|^{2}+\left\|Z_{t+1}^{\varepsilon}-\bar{Z}_{t+1}\right\|^{2}\right] .
\end{aligned}\right.
$$

Thus, there exists $C>0$, such that for any $t \in\{0,1, \ldots, T\}$,

$$
\left\{\begin{aligned}
\mathbb{E}\left|Y_{t}^{\varepsilon}-\bar{Y}_{t}\right|^{2} & \leq C \varepsilon^{2} \mathbb{E}|\Delta v|^{2} \\
\mathbb{E}\left[\left\|Z_{t}^{\varepsilon}-\bar{Z}_{t}\right\|^{2}\right] & \leq C \varepsilon^{2} \mathbb{E}|\Delta v|^{2}
\end{aligned}\right.
$$

This completes the proof.

Let $(\eta, \zeta, V)$ be the solution to the following BS $\Delta \mathrm{E}$,

$$
\left\{\begin{aligned}
\Delta \eta_{t}= & -f_{x}(t+1) \xi_{t+1}-f_{y}(t+1) \eta_{t+1}-\delta_{(t+1) s} f_{u}(t+1) \varepsilon \Delta v \\
& -\sum_{i=1}^{d} f_{z_{i}}(t+1) \zeta_{t+1} e_{i}+\zeta_{t} \Delta W_{t}+\Delta V_{t} \\
\eta_{T}= & 0
\end{aligned}\right.
$$

Notice that $f_{x}(T)=f_{x}\left(T, \bar{X}_{T}, \bar{Y}_{T}, \bar{u}_{T}\right)$ since $f$ is independent of $Z$, also as $f_{y}(T), f_{u}(T)$.

It is easy to check that

$$
\begin{array}{r}
\sup _{0 \leq t \leq T} \mathbb{E}\left|\eta_{t}\right|^{2} \leq C \varepsilon^{2} \mathbb{E}|\Delta v|^{2}, \\
\sup _{0 \leq t \leq T-1} \mathbb{E}\left\|\zeta_{t}\right\|^{2} \leq C \varepsilon^{2} \mathbb{E}|\Delta v|^{2} .
\end{array}
$$

and we have the following result: 
Lemma 3.4 Under Assumption 2.6, we have

$$
\begin{array}{r}
\sup _{0 \leq t \leq T} \mathbb{E}\left|Y_{t}^{\varepsilon}-\bar{Y}_{t}-\eta_{t}\right|^{2}=o\left(\varepsilon^{2}\right), \\
\sup _{0 \leq t \leq T-1} \mathbb{E}\left\|Z_{t}^{\varepsilon}-\bar{Z}_{t}-\zeta_{t}\right\|^{2}=o\left(\varepsilon^{2}\right) .
\end{array}
$$

Proof. When $t=T, Y_{T}^{\varepsilon}-\bar{Y}_{T}-\eta_{T}=0$.

When $t \in\{0,1, \ldots, T-1\}$, we have

$$
\begin{aligned}
& Y_{t}^{\varepsilon}-\bar{Y}_{t}-\eta_{t} \\
& =\mathbb{E}\left[Y_{t+1}^{\varepsilon}+f^{\varepsilon}(t+1)-\bar{Y}_{t+1}-\bar{f}(t+1)-\eta_{t+1}-f_{x}(t+1) \xi_{t+1}\right. \\
& \left.\quad-f_{y}(t+1) \eta_{t+1}-\sum_{i=1}^{d} f_{z_{i}}(t+1) \zeta_{t+1} e_{i}-\delta_{(t+1) s} f_{u}(t+1) \varepsilon \Delta v \mid \mathcal{F}_{t}\right] \\
& =\mathbb{E}\left[Y_{t+1}^{\varepsilon}-\bar{Y}_{t+1}-\eta_{t+1}+\widetilde{f}_{x}(t+1)\left(X_{t+1}^{\varepsilon}-\bar{X}_{t+1}\right)+\widetilde{f}_{y}(t+1)\left(Y_{t+1}^{\varepsilon}-\bar{Y}_{t+1}\right)\right. \\
& \quad+\sum_{i=1}^{d} \tilde{f}_{z_{i}}(t+1)\left(Z_{t+1}^{\varepsilon}-\bar{Z}_{t+1}\right) e_{i}+\delta_{(t+1) s} \tilde{f}_{u}(t+1) \varepsilon \Delta v-f_{x}(t+1) \xi_{t+1} \\
& \left.\quad-f_{y}(t+1) \eta_{t+1}-\sum_{i=1}^{d} f_{z_{i}}(t+1) \zeta_{t+1} e_{i}-\delta_{(t+1) s} f_{u}(t+1) \varepsilon \Delta v \mid \mathcal{F}_{t}\right],
\end{aligned}
$$

where

$$
\widetilde{f}_{\mu}(t)=\int_{0}^{1} f_{\mu}\left(t, \bar{X}_{t}+\lambda\left(X_{t}^{\varepsilon}-\bar{X}_{t}\right), \bar{Y}_{t}+\lambda\left(Y_{t}^{\varepsilon}-\bar{Y}_{t}\right), \bar{Z}_{t}+\lambda\left(Z_{t}^{\varepsilon}-\bar{Z}_{t}\right), \bar{u}_{t}+\lambda\left(u_{t}^{\varepsilon}-\bar{u}_{t}\right) d \lambda\right.
$$

for $\mu=x, y, z_{i}$ and $u$. Then,

$$
\begin{aligned}
& \mathbb{E}\left|Y_{t}^{\varepsilon}-\bar{Y}_{t}-\eta_{t}\right|^{2} \\
& \leq C \mathbb{E}\left[\left|Y_{t+1}^{\varepsilon}-\bar{Y}_{t+1}-\eta_{t+1}\right|^{2}\right. \\
& +\left|\widetilde{f}_{x}(t+1)\left(X_{t+1}^{\varepsilon}-\bar{X}_{t+1}-\xi_{t+1}\right)\right|^{2}+\left|\left[\widetilde{f}_{x}(t+1)-f_{x}(t+1)\right] \xi_{t+1}\right|^{2} \\
& +\left|\widetilde{f}_{y}(t+1)\left(Y_{t+1}^{\varepsilon}-\bar{Y}_{t+1}-\eta_{t+1}\right)\right|^{2}+\left|\left[\widetilde{f}_{y}(t+1)-f_{y}(t+1)\right] \eta_{t+1}\right|^{2} \\
& +\sum_{i=1}^{d}\left|\widetilde{f}_{z_{i}}(t+1)\left(Z_{t+1}^{\varepsilon}-\bar{Z}_{t+1}-\zeta_{t+1}\right) e_{i}\right|^{2}+\sum_{i=1}^{d}\left|\left[\widetilde{f}_{z_{i}}(t+1)-f_{z_{i}}(t+1)\right] \zeta_{t+1} e_{i}\right|^{2} \\
& \left.+\delta_{(t+1) s}\left|\left[\widetilde{f}_{u}(t+1)-f_{u}(t+1)\right] \varepsilon \Delta v\right|^{2}\right]
\end{aligned}
$$


and

$$
\begin{aligned}
& \mathbb{E}\left\|Z_{t}^{\varepsilon}-\bar{Z}_{t}-\zeta_{t}\right\|^{2} \\
& \leq C \mathbb{E}\left[\left|Y_{t+1}^{\varepsilon}-\bar{Y}_{t+1}-\eta_{t+1}\right|^{2}\right. \\
& +\left|\widetilde{f}_{x}(t+1)\left(X_{t+1}^{\varepsilon}-\bar{X}_{t+1}-\xi_{t+1}\right)\right|^{2}+\left|\left[\widetilde{f}_{x}(t+1)-f_{x}(t+1)\right] \xi_{t+1}\right|^{2} \\
& +\left|\widetilde{f}_{y}(t+1)\left(Y_{t+1}^{\varepsilon}-\bar{Y}_{t+1}-\eta_{t+1}\right)\right|^{2}+\left|\left[\widetilde{f}_{y}(t+1)-f_{y}(t+1)\right] \eta_{t+1}\right|^{2} \\
& +\sum_{i=1}^{d}\left|\widetilde{f}_{z_{i}}(t+1)\left(Z_{t+1}^{\varepsilon}-\bar{Z}_{t+1}-\zeta_{t+1}\right) e_{i}\right|^{2}+\sum_{i=1}^{d}\left|\left[\widetilde{f}_{z_{i}}(t+1)-f_{z_{i}}(t+1)\right] \zeta_{t+1} e_{i}\right|^{2} \\
& \left.+\delta_{(t+1) s}\left|\left[\widetilde{f}_{u}(t+1)-f_{u}(t+1)\right] \varepsilon \Delta v\right|^{2}\right] .
\end{aligned}
$$

Notice that $\tilde{f}_{x}(t)-f_{x}(t) \rightarrow 0, \tilde{f}_{y}(t)-f_{y}(t) \rightarrow 0, \tilde{f}_{z_{i}}(t)-f_{z_{i}}(t) \rightarrow 0, \tilde{f}_{u}(t)-f_{u}(t) \rightarrow 0$ as $\varepsilon \rightarrow 0$. We obtain that

$$
\begin{aligned}
\lim _{\varepsilon \rightarrow 0} \frac{1}{\varepsilon^{2}} \mathbb{E}\left|Y_{t}^{\varepsilon}-\bar{Y}_{t}-\eta_{t}\right|^{2} & =0, \\
\lim _{\varepsilon \rightarrow 0} \frac{1}{\varepsilon^{2}} \mathbb{E}\left\|Z_{t}^{\varepsilon}-\bar{Z}_{t}-\zeta_{t}\right\|^{2} & =0 .
\end{aligned}
$$

This completes the proof.

By Lemma 3.2 and Lemma 3.4, we have

$$
\begin{gathered}
J\left(u^{\varepsilon}(\cdot)\right)-J(\bar{u}(\cdot)) \\
=\mathbb{E} \sum_{t=0}^{T-1}\left[\left\langle l_{x}(t), \xi_{t}\right\rangle+\left\langle l_{y}(t), \eta_{t}\right\rangle+\sum_{i=1}^{d}\left\langle l_{z_{i}}(t), \zeta_{t} e_{i}\right\rangle+\delta_{t s}\left\langle l_{u}(s), \varepsilon \Delta v\right\rangle\right] \\
+\mathbb{E}\left\langle h_{x}\left(\bar{X}_{T}\right), \xi_{T}\right\rangle+o(\varepsilon) .
\end{gathered}
$$

Introducing the following adjoint equation:

$$
\left\{\begin{aligned}
\Delta p_{t}= & -b_{x}^{*}(t+1) p_{t+1}-\sum_{i=1}^{d} \sigma_{i x}^{*}(t+1) q_{t+1} e_{i} \\
& +f_{x}^{*}(t+1) k_{t+1}+l_{x}(t+1)+q_{t} \Delta W_{t}+\Delta Q_{t}, \\
\Delta k_{t}= & f_{y}^{*}(t) k_{t}+l_{y}(t)+\sum_{i=1}^{d}\left[f_{z_{i}}^{*}(t) k_{t}+l_{z_{i}}(t)\right] \Delta W_{t}^{i}, \\
p_{T}= & -h_{x}\left(\bar{X}_{T}\right), \\
k_{0}= & 0,
\end{aligned}\right.
$$

where $W$ and $Q$ are square integrable martingale processes and $Q$ is strongly orthogonal to $W$.

Obviously the forward equation in (3.8) admits a unique solution $k \in \mathcal{M}^{2}\left(0, T ; \mathbb{R}^{n}\right)$. Then, based on the solution $k$, according to Theorem 2.4 the backward equation in (3.8) has a unique solution $(p, q, Q) \in$ $\mathcal{M}^{2}\left(0, T ; \mathbb{R}^{m}\right) \times \mathcal{M}^{2}\left(0, T-1 ; \mathbb{R}^{m \times d}\right) \times \mathcal{M}^{2}\left(0, T ; \mathbb{R}^{m}\right)$. So FBS $\Delta \mathrm{E}$ has a unique solution $(p, q, Q, k)$.

We obtain the following maximum principle for the optimal control problem (1.1)-(1.2). 
Define the Hamiltonian function

$$
\begin{array}{r}
H(\omega, t, u, x, y, z, p, q, k)=b^{*}(\omega, t, x, u) p+\sum_{i=1}^{d} \sigma_{i}^{*}(\omega, t, x, u) q e_{i} \\
-f^{*}(\omega, t, x, y, z, u) k-l(\omega, t, x, y, z, u) .
\end{array}
$$

Theorem 3.5 Suppose that Assumption (2.6) holds. Let $\bar{u}$ be an optimal control of the problem (1.1)-(1.2), $(\bar{X}, \bar{Y}, \bar{Z})$ be the corresponding optimal trajectory and $(p, q, k)$ be the solution to the adjoint equation (3.8). Then for any $t \in\{0,1, \ldots, T\}$, for any $v \in U_{t}$, we have

$$
\left\langle H_{u}\left(t, \bar{u}_{t}, \bar{X}_{t}, \bar{Y}_{t}, \bar{Z}_{t}, p_{t}, q_{t}, k_{t}\right), v-\bar{u}_{t}\right\rangle \leq 0, P-a . s . .
$$

Proof. For $t \in\{0,1, \ldots, T-1\}$, we have

$$
\begin{aligned}
& \Delta\left\langle\xi_{t}, p_{t}\right\rangle \\
= & \left\langle\xi_{t+1}, \Delta p_{t}\right\rangle+\left\langle\Delta \xi_{t}, p_{t}\right\rangle \\
= & \left\langle\xi_{t+1},-b_{x}^{*}(t+1) p_{t+1}-\sum_{i=1}^{d} \sigma_{i x}^{*}(t+1) q_{t+1} e_{i}+f_{x}^{*}(t+1) k_{t+1}+l_{x}(t+1)\right\rangle \\
& +\left\langle\sum_{j=1}^{d}\left[\sigma_{j x}(t) \xi_{t}+\delta_{t s} \varepsilon \sigma_{j u}(t) \Delta v\right] \Delta W_{t}^{j}, q_{t} \Delta W_{t}\right\rangle+\left\langle b_{x}(t) \xi_{t}+\delta_{t s} b_{u}(t) \varepsilon \Delta v, p_{t}\right\rangle+\Phi_{t},
\end{aligned}
$$

where

$$
\begin{aligned}
\Phi_{t}= & \left\langle\xi_{t}+b_{x}(t) \xi_{t}+\delta_{t s} b_{u}(t) \varepsilon \Delta v, q_{t} \Delta W_{t}\right\rangle+\left\langle\sum_{i=1}^{d}\left[\sigma_{i x}(t) \xi_{t}+\delta_{t s} \varepsilon \sigma_{i u}(t) \Delta v\right] \Delta W_{t}^{i}, p_{t}\right\rangle \\
& +\left\langle\xi_{t}+b_{x}(t) \xi_{t}+\delta_{t s} b_{u}(t) \varepsilon \Delta v, \Delta Q_{t}\right\rangle+\left\langle\sum_{j=1}^{d}\left[\sigma_{j x}(t) \xi_{t}+\delta_{t s} \varepsilon \sigma_{j u}(t) \Delta v\right] \Delta W_{t}^{j}, \Delta Q_{t}\right\rangle .
\end{aligned}
$$

It is obvious that $\mathbb{E}\left[\Phi_{t}\right]=0$. We have

$$
\begin{aligned}
\mathbb{E}\left\langle\sum_{j=1}^{d} \sigma_{j x}(t) \xi_{t} \Delta W_{t}^{j}, q_{t} \Delta W_{t}\right\rangle & =\mathbb{E}\left\langle\sum_{j=1}^{d} \sigma_{j x}(t) \xi_{t} \Delta W_{t}^{j}, \sum_{i=1}^{d} q_{t} e_{i} \Delta W_{t}^{i}\right\rangle \\
& =\mathbb{E}\left[\sum_{i=1}^{d}\left\langle\xi_{t}, \sum_{j=1}^{d} \sigma_{j x}^{*}(t) q_{t} e_{i} \mathbb{E}\left[\Delta W_{t}^{i} \Delta W_{t}^{j} \mid \mathcal{F}_{t}\right]\right\rangle\right] \\
& =\mathbb{E}\left[\sum_{i=1}^{d}\left\langle\xi_{t}, \sigma_{i x}^{*}(t) q_{t} e_{i}\right\rangle\right]
\end{aligned}
$$

and

$$
\mathbb{E}\left\langle\sum_{j=1}^{d} \delta_{t s} \varepsilon \sigma_{j u}(t) \Delta v \Delta W_{t}^{j}, q_{t} \Delta W_{t}\right\rangle=\mathbb{E}\left[\delta_{t s} \varepsilon \sum_{j=1}^{d}\left\langle\sigma_{j u}(t) \Delta v, q_{t} e_{j}\right\rangle\right] .
$$

Similarly, it can be shown that for $t \in\{0,1, \ldots, T-1\}$, we have

$$
\begin{aligned}
& \Delta\left\langle\eta_{t}, k_{t}\right\rangle \\
= & \left\langle-f_{x}(t+1) \xi_{t+1}-f_{y}(t+1) \eta_{t+1}-\sum_{i=1}^{d} f_{z_{i}}(t+1) \zeta_{t+1} e_{i}-\delta_{(t+1) s} f_{u}(t+1) \varepsilon \Delta v, k_{t+1}\right\rangle \\
& +\left\langle\zeta_{t} \Delta W_{t}, \sum_{i=1}^{d}\left[f_{z_{i}}^{*}(t) k_{t}+l_{z_{i}}(t)\right] \Delta W_{t}^{i}\right\rangle+\left\langle\eta_{t}, f_{y}^{*}(t) k_{t}+l_{y}(t)\right\rangle+\Psi_{t},
\end{aligned}
$$


where

$$
\begin{aligned}
\Psi_{t}= & \left\langle\zeta_{t} \Delta W_{t}, k_{t}+f_{y}^{*}(t) k_{t}+l_{y}(t)\right\rangle+\left\langle\eta_{t}, \sum_{i=1}^{d}\left[f_{z_{i}}^{*}(t) k_{t}+l_{z_{i}}(t)\right] \Delta W_{t}^{i}\right\rangle \\
& +\left\langle k_{t}+f_{y}^{*}(t) k_{t}+l_{y}(t), \Delta V_{t}\right\rangle+\left\langle\sum_{i=1}^{d}\left[f_{z_{i}}^{*}(t) k_{t}+l_{z_{i}}(t)\right] \Delta W_{t}^{i}, \Delta V_{t}\right\rangle .
\end{aligned}
$$

It is easy to check that

$$
\begin{aligned}
\mathbb{E}\left\langle\zeta_{t} \Delta W_{t}, \sum_{i=1}^{d} f_{z_{i}}^{*}(t) k_{t} \Delta W_{t}^{i}\right\rangle & =\mathbb{E}\left[\sum_{i=1}^{d}\left\langle f_{z_{i}}(t) \zeta_{t} e_{i}, k_{t}\right\rangle\right], \\
\mathbb{E}\left\langle\zeta_{t} \Delta W_{t}, \sum_{i=1}^{d} l_{z_{i}}(t) \Delta W_{t}^{i}\right\rangle & =\mathbb{E}\left[\sum_{i=1}^{d}\left\langle l_{z_{i}}(t), \zeta_{t} e_{i}\right\rangle\right] .
\end{aligned}
$$

Then we have

$$
\begin{aligned}
& \mathbb{E}\left[\Delta\left(\left\langle\xi_{t}, p_{t}\right\rangle+\left\langle\eta_{t}, k_{t}\right\rangle\right)\right] \\
= & \mathbb{E}\left[\left\langle-b_{x}(t+1) \xi_{t+1}, p_{t+1}\right\rangle+\left\langle b_{x}(t) \xi_{t}, p_{t}\right\rangle\right. \\
- & \sum_{i=1}^{d}\left\langle\xi_{t+1}, \sigma_{i x}^{*}(t+1) q_{t+1} e_{i}\right\rangle+\sum_{i=1}^{d}\left\langle\xi_{t}, \sigma_{i x}^{*}(t) q_{t} e_{i}\right\rangle \\
- & \left\langle f_{y}(t+1) \eta_{t+1}, k_{t+1}\right\rangle+\left\langle f_{y}(t) \eta_{t}, k_{t}\right\rangle \\
& -\sum_{i=1}^{d}\left\langle f_{z_{i}}(t+1) \zeta_{t+1} e_{i}, k_{t+1}\right\rangle+\sum_{i=1}^{d}\left\langle f_{z_{i}}(t) \zeta_{t} e_{i}, k_{t}\right\rangle \\
+ & \left\langle l_{x}(t+1), \xi_{t+1}\right\rangle+\left\langle\eta_{t}, l_{y}(t)\right\rangle+\sum_{i=1}^{d}\left\langle l_{z_{i}}(t), \zeta_{t} e_{i}\right\rangle \\
& +\varepsilon\left\langle\delta_{t s} b_{u}(t) \Delta v, p_{t}\right\rangle+\delta_{t s} \varepsilon \sum_{i=1}^{d}\left\langle\sigma_{i u}(t) \Delta v, q_{t} e_{i}\right\rangle \\
& \left.-\varepsilon\left\langle\delta_{(t+1) s} f_{u}(t+1) \Delta v, k_{t+1}\right\rangle\right] .
\end{aligned}
$$

Therefore,

$$
\begin{aligned}
& -\mathbb{E}\left\langle h_{x}\left(\bar{X}_{T}\right), \xi_{T}\right\rangle \\
= & \mathbb{E}\left[\left\langle\xi_{T}, p_{T}\right\rangle+\left\langle\eta_{T}, k_{T}\right\rangle-\left\langle\xi_{0}, p_{0}\right\rangle-\left\langle\eta_{0}, k_{0}\right\rangle\right] \\
= & \sum_{t=0}^{T-1} \mathbb{E} \Delta\left(\left\langle\xi_{t}, p_{t}\right\rangle+\left\langle\eta_{t}, k_{t}\right\rangle\right) \\
= & \mathbb{E}\left[\left\langle b_{x}(0) \xi_{0}, p_{0}\right\rangle+\sum_{i=1}^{d}\left\langle\xi_{0}, \sigma_{i x}^{*}(0) q_{0} e_{i}\right\rangle+\left\langle f_{y}(0) \eta_{0}, k_{0}\right\rangle+\sum_{i=1}^{d}\left\langle f_{z_{i}}(0) \zeta_{0} e_{i}, k_{0}\right\rangle\right] \\
& +\sum_{t=0}^{T-1} \mathbb{E}\left[\left\langle l_{x}(t), \xi_{t}\right\rangle+\left\langle l_{y}(t), \eta_{t}\right\rangle+\sum_{i=1}^{d}\left\langle l_{z_{i}}(t), \zeta_{t} e_{i}\right\rangle\right] \\
& +\sum_{t=0}^{T} \delta_{t s} \varepsilon \mathbb{E}\left[\left\langle b_{u}^{*}(t) p_{t}, \Delta v\right\rangle+\sum_{i=1}^{d}\left\langle\sigma_{i u}^{*}(t) q_{t} e_{i}, \Delta v\right\rangle-\left\langle f_{u}^{*}(t) k_{t}, \Delta v\right\rangle\right] .
\end{aligned}
$$


Since $\xi_{0}=0$ and $k_{0}=0$, we deduce

$$
\begin{aligned}
& \mathbb{E} \sum_{t=0}^{T-1}\left[\left\langle l_{x}(t), \xi_{t}\right\rangle+\left\langle l_{y}(t), \eta_{t}\right\rangle+\sum_{i=1}^{d}\left\langle l_{z_{i}}(t), \zeta_{t} e_{i}\right\rangle\right]+\mathbb{E}\left\langle h_{x}\left(\bar{X}_{T}\right), \xi_{T}\right\rangle \\
= & -\varepsilon \mathbb{E}\left[\left\langle b_{u}^{*}(s) p_{s}+\sum_{i=1}^{d} \sigma_{i u}^{*}(s) q_{t} e_{i}-f_{u}^{*}(s) k_{s}, \Delta v\right\rangle\right] .
\end{aligned}
$$

By $\lim _{\varepsilon \rightarrow 0} \frac{1}{\varepsilon}\left[J\left(u^{\varepsilon}(\cdot)\right)-J(\bar{u}(\cdot))\right] \geq 0$, we obtain

$$
\mathbb{E}\left[\left\langle b_{u}^{*}(s) p_{s}+\sum_{i=1}^{d} \sigma_{i u}^{*}(s) q_{s} e_{i}-f_{u}^{*}(s) k_{s}-l_{u}(s), \Delta v\right\rangle\right] \leq 0 .
$$

Thus, it is easy to obtain equation (3.9) since $s$ is taking arbitrarily. This completes the proof.

Remark 3.6 In the introduction we point out that we need a reasonable representation of the product rule. When we calculate $\Delta\left\langle\xi_{t}, p_{t}\right\rangle$ in (3.10), $\Delta\left\langle\xi_{t}, p_{t}\right\rangle$ is represented as $\left\langle\xi_{t+1}, \cdots\right\rangle+\cdots$. Combining the formulation of the BSAE mentioned in the introduction, this representation will lead to the terms such as $\left\langle\square_{t}, \diamond_{t}\right\rangle-$ $\left\langle\square_{t+1}, \diamond_{t+1}\right\rangle$ in (3.11). By summing and rearranging these terms in (3.12), we obtain the dual relation (3.13).

When $g \equiv 0$ and $f \equiv 0$, our control system (1.1)-(1.2) degenerates to the classical discrete control system which only contains a forward stochastic difference equation as in 14. For this special case, the adjoint equation becomes

$$
\left\{\begin{aligned}
\Delta p_{t} & =-b_{x}^{*}(t+1) p_{t+1}-\sum_{i=1}^{d} \sigma_{i x}^{*}(t+1) q_{t+1} e_{i}+l_{x}(t+1)+q_{t} \Delta W_{t}+\Delta Q_{t} \\
p_{T} & =-h_{x}\left(\bar{X}_{T}\right)
\end{aligned}\right.
$$

and the Hamiltonian function becomes

$$
H(\omega, t, u, x, p, q)=b^{*}(\omega, t, x, u) p+\sum_{i=1}^{d} \sigma_{i}^{*}(\omega, t, x, u) q e_{i}-l(\omega, t, x, u) .
$$

The adjoint equation has the following explicit solution

$$
\left\{\begin{aligned}
p_{T-1} & =-\mathbb{E}\left[h_{x}\left(\bar{X}_{T}\right) \mid \mathcal{F}_{T-1}\right], \\
q_{T-1} & =-\mathbb{E}\left[h_{x}\left(\bar{X}_{T}\right)\left(\Delta W_{t}\right)^{*} \mid \mathcal{F}_{T-1}\right], \\
p_{t} & =\mathbb{E}\left[\left[I+b_{x}^{*}(t+1)\right] p_{t+1}-l_{x}(t+1)+\sum_{i=1}^{d} \sigma_{i x}^{*}(t+1) q_{t+1} e_{i} \mid \mathcal{F}_{t}\right], \\
q_{t} & =\mathbb{E}\left[\left[\left[I+b_{x}^{*}(t+1)\right] p_{t+1}-l_{x}(t+1)+\sum_{i=1}^{d} \sigma_{i x}^{*}(t+1) q_{t+1} e_{i}\right]\left(\Delta W_{t}\right)^{*} \mid \mathcal{F}_{t}\right] .
\end{aligned}\right.
$$

which coincides with the results in [14]. 


\section{Maximum principle for the fully coupled FBS $\Delta \mathrm{E}$ system}

In this section we suppose $W$ to be one-dimensional driving process. Let $\bar{u}=\left\{\bar{u}_{t}\right\}_{t=0}^{T}$ be the optimal control for the control problem (1.3)-(1.4) and $(\bar{X}, \bar{Y}, \bar{Z})$ be the corresponding optimal trajectory. Note that the existence and uniqueness of $(\bar{X}, \bar{Y}, \bar{Z})$ is guaranteed by the results in [15]. The perturbed control $u^{\varepsilon}$ is the same as (3.1) and we denote by $\left(X^{\varepsilon}, Y^{\varepsilon}, Z^{\varepsilon}\right)$ the corresponding trajectory.

Let

$$
\widehat{X}_{t}=X_{t}^{\varepsilon}-\bar{X}_{t}, \widehat{Y}_{t}=Y_{t}^{\varepsilon}-\bar{Y}_{t}, \widehat{Z}_{t}=Z_{t}^{\varepsilon}-\bar{Z}_{t}, \widehat{N}_{t}=N_{t}^{\varepsilon}-\bar{N}_{t}
$$

Using the similar notations (3.2) in section 3 , we have

$$
\left\{\begin{aligned}
\Delta \widehat{X}_{t} & =b^{\varepsilon}(t)-\bar{b}(t)+\left(\sigma^{\varepsilon}(t)-\bar{\sigma}(t)\right) \Delta W_{t}, \\
\Delta \widehat{Y}_{t} & =-f^{\varepsilon}(t+1)+\bar{f}(t+1)+\widehat{Z}_{t} \Delta W_{t}+\Delta \widehat{N}_{t} \\
\widehat{X}_{0} & =0 \\
\widehat{Y}_{T} & =0 .
\end{aligned}\right.
$$

Lemma 4.1 Under Assumption 2.6 and Assumption 2.7, we have

$$
\mathbb{E}\left(\sum_{t=0}^{T}\left|\widehat{X}_{t}\right|^{2}+\sum_{t=0}^{T}\left|\widehat{Y}_{t}\right|^{2}+\sum_{t=0}^{T-1}\left|\widehat{Z}_{t}\right|^{2}\right) \leq C \varepsilon^{2} \mathbb{E}|\Delta v|^{2} .
$$

Proof. By (4.1),

$$
\begin{aligned}
0= & \mathbb{E}\left\langle\widehat{X}_{T}, \widehat{Y}_{T}\right\rangle-\mathbb{E}\left\langle\widehat{X}_{0}, \widehat{Y}_{0}\right\rangle \\
= & \mathbb{E} \sum_{t=0}^{T-1} \Delta\left\langle\widehat{X}_{t}, \widehat{Y}_{t}\right\rangle \\
= & \mathbb{E} \sum_{t=0}^{T}\left[\left\langle\widehat{X}_{t},-f^{\varepsilon}(t)+\bar{f}(t)\right\rangle+\left\langle\widehat{Y}_{t}, b^{\varepsilon}(t)-\bar{b}(t)\right\rangle+\left\langle\widehat{Z}_{t}, \sigma^{\varepsilon}(t)-\bar{\sigma}(t)\right\rangle\right] \\
= & \mathbb{E} \sum_{t=1}^{T-1}\left\langle A\left(t, \lambda_{t}^{\varepsilon} ; u_{t}^{\varepsilon}\right)-A\left(t, \bar{\lambda}_{t} ; u_{t}^{\varepsilon}\right), \widehat{\lambda}_{t}\right\rangle \\
& +\mathbb{E}\left\langle\widehat{X}_{T},-f^{\varepsilon}(T)+\widetilde{f}^{\varepsilon}(T)\right\rangle+\left\langle\widehat{Y}_{0}, b^{\varepsilon}(0)-\widetilde{b}^{\varepsilon}(0)\right\rangle+\left\langle\widehat{Z}_{0}, \sigma^{\varepsilon}(0)-\widetilde{\sigma}^{\varepsilon}(0)\right\rangle \\
& +\mathbb{E} \sum_{t=0}^{T}\left[\left\langle\widehat{X}_{t},-\widetilde{f}^{\varepsilon}(t)+\bar{f}(t)\right\rangle+\left\langle\widehat{Y}_{t}, \widetilde{b}^{\varepsilon}(t)-\bar{b}(t)\right\rangle+\left\langle\widehat{Z}_{t}, \widetilde{\sigma}^{\varepsilon}(t)-\bar{\sigma}(t)\right\rangle\right] \\
= & \mathbb{E} \sum_{t=1}^{T-1}\left\langle A\left(t, \lambda_{t}^{\varepsilon} ; u_{t}^{\varepsilon}\right)-A\left(t, \bar{\lambda}_{t} ; u_{t}^{\varepsilon}\right), \widehat{\lambda}_{t}\right\rangle \\
& +\mathbb{E}\left\langle\widehat{X}_{T},-f^{\varepsilon}(T)+\widetilde{f}^{\varepsilon}(T)\right\rangle+\left\langle\widehat{Y}_{0}, b^{\varepsilon}(0)-\widetilde{b}^{\varepsilon}(0)\right\rangle+\left\langle\widehat{Z}_{0}, \sigma^{\varepsilon}(0)-\widetilde{\sigma}^{\varepsilon}(0)\right\rangle \\
& +\mathbb{E}\left[\left\langle\widehat{X}_{s},-\widetilde{f}^{\varepsilon}(s)+\bar{f}(s)\right\rangle+\left\langle\widehat{Y}_{s}, \widetilde{b}^{\varepsilon}(s)-\bar{b}(s)\right\rangle+\left\langle\widehat{Z}_{s}, \widetilde{\sigma}^{\varepsilon}(s)-\bar{\sigma}(s)\right\rangle\right]
\end{aligned}
$$


By the monotone condition, we obtain

$$
\begin{aligned}
& \mathbb{E}\left[\left\langle\widehat{X}_{s},-\widetilde{f}^{\varepsilon}(s)+\bar{f}(s)\right\rangle+\left\langle\widehat{Y}_{s}, \widetilde{b}^{\varepsilon}(s)-\bar{b}(s)\right\rangle+\left\langle\widehat{Z}_{s}, \tilde{\sigma}^{\varepsilon}(s)-\bar{\sigma}(s)\right\rangle\right] \\
\geq & \alpha \mathbb{E}\left[\sum_{t=0}^{T}\left|\widehat{X}_{t}\right|^{2}+\sum_{t=0}^{T}\left|\widehat{Y}_{t}\right|^{2}+\sum_{t=0}^{T-1}\left|\widehat{Z}_{t}\right|^{2}\right] .
\end{aligned}
$$

On the other hand,

$$
\begin{aligned}
& \mathbb{E}\left\langle\widehat{X}_{s},-\widetilde{f}^{\varepsilon}(s)+\bar{f}(s)\right\rangle \\
\leq & \frac{\alpha}{2} \mathbb{E}\left|\widehat{X}_{s}\right|^{2}+\frac{1}{2 \alpha} \mathbb{E}\left|\bar{f}(s)-\widetilde{f}^{\varepsilon}(s)\right|^{2} \\
\leq & \frac{\alpha}{2} \mathbb{E}\left|\widehat{X}_{s}\right|^{2}+\frac{C}{2 \alpha} \varepsilon^{2} \mathbb{E}|\Delta v|^{2}
\end{aligned}
$$

and similarly,

$$
\begin{aligned}
& \mathbb{E}\left[\left\langle\widehat{X}_{s},-\widetilde{f}^{\varepsilon}(s)+\bar{f}(s)\right\rangle+\left\langle\widehat{Y}_{s}, \widetilde{b}^{\varepsilon}(s)-\bar{b}(s)\right\rangle+\left\langle\widehat{Z}_{s}, \widetilde{\sigma}^{\varepsilon}(s)-\bar{\sigma}(s)\right\rangle\right] \\
\leq & \frac{\alpha}{2} \mathbb{E}\left[\left|\widehat{X}_{s}\right|^{2}+\left|\widehat{Y}_{s}\right|^{2}+\left|\widehat{Z}_{s}\right|^{2}\right]+C \varepsilon^{2} \mathbb{E}|\Delta v|^{2} .
\end{aligned}
$$

Combining (4.3) and (4.4), we have

$$
\mathbb{E}\left[\sum_{t=0}^{T}\left|\widehat{X}_{t}\right|^{2}+\sum_{t=0}^{T}\left|\widehat{Y}_{t}\right|^{2}+\sum_{t=0}^{T-1}\left|\widehat{Z}_{t}\right|^{2}\right] \leq C \varepsilon^{2} \mathbb{E}|\Delta v|^{2} .
$$

This completes the proof.

Next we introduce the following variational equation:

$$
\left\{\begin{aligned}
\Delta \xi_{t}= & b_{x}(t) \xi_{t}+b_{y}(t) \eta_{t}+b_{z}(t) \zeta_{t}+\delta_{t s} b_{u}(t) \varepsilon \Delta v \\
& +\left[\sigma_{x}(t) \xi_{t}+\sigma_{y}(t) \eta_{t}+\sigma_{z}(t) \zeta_{t}+\delta_{t s} \varepsilon \sigma_{u}(t) \Delta v\right] \Delta W_{t} \\
\Delta \eta_{t}= & -f_{x}(t+1) \xi_{t+1}-f_{y}(t+1) \eta_{t+1}-f_{z}(t+1) \zeta_{t+1} \\
& -\delta_{(t+1) s} f_{u}(t+1) \varepsilon \Delta v+\zeta_{t} \Delta W_{t}+\Delta V_{t} \\
\xi_{0}= & 0 \\
\eta_{T}= & 0
\end{aligned}\right.
$$

By Assumption 2.6 and Assumption 2.7, when $t \in\{1, \ldots, T-1\}$,

$$
\left(\begin{array}{ccc}
-f_{x}(t) & -f_{y}(t) & -f_{z}(t) \\
b_{x}(t) & b_{y}(t) & b_{z}(t) \\
\sigma_{x}(t) & \sigma_{y}(t) & \sigma_{z}(t)
\end{array}\right) \leq-\alpha I_{3 n}, P-a . s .
$$


when $t=0$,

$$
\left(\begin{array}{cc}
b_{y}(0) & b_{z}(0) \\
\sigma_{y}(0) & \sigma_{z}(0)
\end{array}\right) \leq-\alpha I_{2 n}, P-a . s .
$$

when $t=T$,

$$
-f_{x}(T) \leq-\alpha I_{n}, P-a . s . .
$$

Thus, the coefficients of (4.5) satisfy the monotone condition and there exists a unique solution $(\xi, \eta, \zeta, V)$ to (4.5). Similar to the proof of Lemma 4.1, we have

$$
\mathbb{E}\left[\sum_{t=0}^{T}\left|\xi_{t}\right|^{2}+\sum_{t=0}^{T}\left|\eta_{t}\right|^{2}+\sum_{t=0}^{T-1}\left|\zeta_{t}\right|^{2}\right] \leq C \varepsilon^{2} \mathbb{E}|\Delta v|^{2}
$$

Define

$$
\widetilde{\varphi}_{\mu}(t)=\int_{0}^{1} \varphi_{\mu} t, \bar{X}_{t}+\lambda\left(X_{t}^{\varepsilon}-\bar{X}_{t}\right), \bar{Y}_{t}+\lambda\left(Y_{t}^{\varepsilon}-\bar{Y}_{t}\right), \bar{Z}_{t}+\lambda\left(Z_{t}^{\varepsilon}-\bar{Z}_{t}\right), \bar{u}_{t}+\lambda\left(u_{t}^{\varepsilon}-\bar{u}_{t}\right) d \lambda
$$

where $\varphi=b, \sigma_{i}, g, f, l, h$ and $\mu=x, y, z_{i}$ and $u$.

Lemma 4.2 Under Assumption [2.6] and Assumption 2.7, we have

$$
\mathbb{E}\left[\sum_{t=0}^{T}\left|\widehat{X}_{t}-\xi_{t}\right|^{2}+\sum_{t=0}^{T}\left|\widehat{Y}_{t}-\eta_{t}\right|^{2}+\sum_{t=0}^{T-1}\left|\widehat{Z}_{t}-\zeta_{t}\right|^{2}\right]=o\left(\varepsilon^{2}\right) .
$$

Proof. Note that

$$
\begin{aligned}
& \varphi^{\varepsilon}(t)-\bar{\varphi}(t) \\
& =\widetilde{\varphi}_{x}(t)\left(X_{t}^{\varepsilon}-\bar{X}_{t}\right)+\widetilde{\varphi}_{y}(t)\left(Y_{t}^{\varepsilon}-\bar{Y}_{t}\right)+\widetilde{\varphi}_{z}(t)\left(Z_{t}^{\varepsilon}-\bar{Z}_{t}\right)+\delta_{t s} \widetilde{\varphi}_{u}(t) \varepsilon \Delta v .
\end{aligned}
$$

Set

$$
\widetilde{X}_{t}=\widehat{X}_{t}-\xi_{t}, \widetilde{Y}_{t}=\widehat{Y}_{t}-\eta_{t}, \widetilde{Z}_{t}=\widehat{Z}_{t}-\zeta_{t}, \widetilde{N}_{t}=\widehat{N}_{t}-V_{t}
$$

Then,

$$
\left\{\begin{aligned}
\Delta \widetilde{X}_{t}= & b_{x}(t) \widetilde{X}_{t}+b_{y}(t) \widetilde{Y}_{t}+b_{z}(t) \widetilde{Z}_{t}+\Lambda_{1}(t) \\
& +\left[\sigma_{x}(t) \widetilde{X}_{t}+\sigma_{y}(t) \widetilde{Y}_{t}+\sigma_{z}(t) \widetilde{Z}_{t}+\Lambda_{2}(t)\right] \Delta W_{t}, \\
\Delta \widetilde{Y}_{t}= & -f_{x}(t+1) \widetilde{X}_{t+1}-f_{y}(t+1) \widetilde{Y}_{t+1}-f_{z}(t+1) \widetilde{Z}_{t+1} \\
& -\Lambda_{3}(t+1)+\widetilde{Z}_{t} \Delta W_{t}+\Delta \widetilde{N}_{t}, \\
\widetilde{X}_{0}= & 0, \\
\widetilde{Y}_{T}= & 0,
\end{aligned}\right.
$$


where

$$
\begin{aligned}
\Lambda_{1}(t) & =\left(\widetilde{b}_{x}(t)-b_{x}(t)\right) \widehat{X}_{t}+\left(\widetilde{b}_{y}(t)-b_{y}(t)\right) \widehat{Y}_{t} \\
& +\left(\widetilde{b}_{z}(t)-b_{z}(t)\right) \widehat{Z}_{t}+\delta_{t s}\left(\widetilde{b}_{u}(t)-b_{u}(t)\right) \varepsilon \Delta v, \\
\Lambda_{2}(t) & =\left(\widetilde{\sigma}_{x}(t)-\sigma_{x}(t)\right) \widehat{X}_{t}+\left(\widetilde{\sigma}_{y}(t)-\sigma_{y}(t)\right) \widehat{Y}_{t} \\
& +\left(\widetilde{\sigma}_{z}(t)-\sigma_{z}(t)\right) \widehat{Z}_{t}+\delta_{t s}\left(\widetilde{\sigma}_{u}(t)-\sigma_{u}(t)\right) \varepsilon \Delta v, \\
\Lambda_{3}(t) & =-\left(\widetilde{f}_{x}(t)-f_{x}(t)\right) \widehat{X}_{t}-\left(\widetilde{f}_{y}(t)-f_{y}(t)\right) \widehat{Y}_{t} \\
& -\left(\widetilde{f}_{z}(t)-f_{z}(t)\right) \widehat{Z}_{t}-\delta_{t s}\left(\widetilde{f}_{u}(t)-f_{u}(t)\right) \varepsilon \Delta v .
\end{aligned}
$$

According to (4.10),

$$
\begin{aligned}
0= & \mathbb{E}\left\langle\widetilde{X}_{T}, \widetilde{Y}_{T}\right\rangle-\mathbb{E}\left\langle\widetilde{X}_{0}, \widetilde{Y}_{0}\right\rangle \\
= & \mathbb{E} \sum_{t=0}^{T-1} \Delta\left\langle\widetilde{X}_{t}, \widetilde{Y}_{t}\right\rangle \\
= & \mathbb{E} \sum_{t=0}^{T}\left[\left\langle\widetilde{X}_{t},-f_{\lambda}(t) \widetilde{\lambda}_{t}\right\rangle+\left\langle\widetilde{Y}_{t}, b_{\lambda}(t) \widetilde{\lambda}_{t}\right\rangle+\left\langle\widetilde{Z}_{t}, \sigma_{\lambda}(t) \widetilde{\lambda}_{t}\right\rangle\right] \\
& +\mathbb{E} \sum_{t=0}^{T}\left[\left\langle\widetilde{X}_{t},-\Lambda_{3}(t)\right\rangle+\left\langle\widetilde{Y}_{t}, \Lambda_{1}(t)\right\rangle+\left\langle\widetilde{Z}_{t}, \Lambda_{2}(t)\right\rangle\right]
\end{aligned}
$$

where

$$
\begin{aligned}
\widetilde{\lambda}_{t} & =\left(\widetilde{X}_{t}^{*}, \widetilde{Y}_{t}^{*}, \widetilde{Z}_{t}^{*}\right)^{*}, \\
b_{\lambda}(t) & =\left(b_{x}(t), b_{y}(t), b_{z}(t)\right), \\
\sigma_{\lambda}(t) & =\left(\sigma_{x}(t), \sigma_{y}(t), \sigma_{z}(t)\right), \\
f_{\lambda}(t) & =\left(f_{x}(t), f_{y}(t), f_{z}(t)\right) .
\end{aligned}
$$

Combining (4.6), (4.7) and (4.8), we have

$$
\begin{aligned}
& \mathbb{E} \sum_{t=0}^{T}\left[\left\langle\widetilde{X}_{t},-\Lambda_{3}(t)\right\rangle+\left\langle\widetilde{Y}_{t}, \Lambda_{1}(t)\right\rangle+\left\langle\widetilde{Z}_{t}, \Lambda_{2}(t)\right\rangle\right] \\
\geq & \alpha \mathbb{E}\left[\sum_{t=0}^{T}\left|\widetilde{X}_{t}\right|^{2}+\sum_{t=0}^{T}\left|\widetilde{Y}_{t}\right|^{2}+\sum_{t=0}^{T-1}\left|\widetilde{Z}_{t}\right|^{2}\right] .
\end{aligned}
$$

Note that

$$
\begin{aligned}
& \mathbb{E}\left\langle\widetilde{X}_{t},-\Lambda_{3}(t)\right\rangle \\
= & \mathbb{E}\left\langle\widetilde{X}_{t},\left(\widetilde{f}_{x}(t)-f_{x}(t)\right) \widehat{X}_{t}\right\rangle+\mathbb{E}\left\langle\widetilde{X}_{t},\left(\widetilde{f}_{y}(t)-f_{y}(t)\right) \widehat{Y}_{t}\right\rangle \\
& +\mathbb{E}\left\langle\widetilde{X}_{t},\left(\widetilde{f}_{z}(t)-f_{z}(t)\right) \widehat{Z}_{t}\right\rangle+\mathbb{E}\left\langle\widetilde{X}_{t}, \delta_{t s}\left(\widetilde{f}_{u}(t)-f_{u}(t)\right) \varepsilon \Delta v\right\rangle \\
\leq & \frac{\alpha}{2} \mathbb{E}\left|\widetilde{X}_{t}\right|^{2}+\frac{2}{\alpha} \mathbb{E}\left[\left\|\widetilde{f}_{x}(t)-f_{x}(t)\right\|^{2}\left|\widehat{X}_{t}\right|^{2}+\left\|\widetilde{f}_{y}(t)-f_{y}(t)\right\|^{2}\left|\widehat{Y}_{t}\right|^{2}\right] \\
& +\frac{2}{\alpha} \mathbb{E}\left[\left\|\widetilde{f}_{z}(t)-f_{z}(t)\right\|^{2}\left|\widehat{Z}_{t}\right|^{2}+\delta_{t s} \varepsilon^{2}\left\|\widetilde{f}_{u}(t)-f_{u}(t)\right\|^{2}|\Delta v|^{2}\right] .
\end{aligned}
$$


When $\varepsilon \rightarrow 0,\left\|\tilde{f}_{\mu}(t)-f_{\mu}(t)\right\| \rightarrow 0$ for $\mu=x, y, z$ and $u$. Then, by Lemma 4.1

$$
\mathbb{E}\left\langle\widetilde{X}_{t},-\Lambda_{3}(t)\right\rangle \leq \frac{\alpha}{2} \mathbb{E}\left|\widetilde{X}_{t}\right|^{2}+o\left(\varepsilon^{2}\right)
$$

Similar results hold for the other terms in (4.11). Finally, we have

$$
\mathbb{E}\left[\sum_{t=0}^{T}\left|\widetilde{X}_{t}\right|^{2}+\sum_{t=0}^{T}\left|\widetilde{Y}_{t}\right|^{2}+\sum_{t=0}^{T-1}\left|\widetilde{Z}_{t}\right|^{2}\right] \leq o\left(\varepsilon^{2}\right) .
$$

This completes the proof.

By Lemma 4.2, we obtain

$$
\begin{aligned}
& J\left(u^{\varepsilon}(\cdot)\right)-J(\bar{u}(\cdot)) \\
= & \mathbb{E} \sum_{t=0}^{T-1}\left[\left\langle l_{x}(t), \xi_{t}\right\rangle+\left\langle l_{y}(t), \eta_{t}\right\rangle+\left\langle l_{z}(t), \zeta_{t}\right\rangle+\delta_{t s}\left\langle l_{u}(s), \varepsilon \Delta v\right\rangle\right]+\mathbb{E}\left\langle h_{x}\left(\bar{X}_{T}\right), \xi_{T}\right\rangle+o(\varepsilon) .
\end{aligned}
$$

Introduce the following adjoint equation:

$$
\left\{\begin{aligned}
\Delta p_{t}= & -b_{x}^{*}(t+1) p_{t+1}-\sigma_{x}^{*}(t+1) q_{t+1} \\
& +f_{x}^{*}(t+1) k_{t+1}+l_{x}(t+1)+q_{t} \Delta W_{t}+\Delta Q_{t}, \\
\Delta k_{t}= & f_{y}^{*}(t) k_{t}-b_{y}^{*}(t) p_{t}-\sigma_{y}^{*}(t) q_{t}+l_{y}(t) \\
& +\left[f_{z}^{*}(t) k_{t}-b_{z}^{*}(t) p_{t}-\sigma_{z}^{*}(t) q_{t}+l_{z}(t)\right] \Delta W_{t}, \\
p_{T}= & -h_{x}\left(\bar{X}_{T}\right) \\
k_{0}= & 0 .
\end{aligned}\right.
$$

Define the Hamiltonian function as follows:

$$
\begin{array}{r}
H(\omega, t, u, x, y, z, p, q, k)=b^{*}(\omega, t, x, y, z, u) p+\sum_{i=1}^{d} \sigma_{i}^{*}(\omega, t, x, y, z, u) q e_{i} \\
-f^{*}(\omega, t, x, y, z, u) k-l(\omega, t, x, y, z, u)
\end{array}
$$

Theorem 4.3 Suppose that Assumption[2.6 and Assumption 2.7 hold. Let $\bar{u}$ be an optimal control for (1.3)(1.3), $(\bar{X}, \bar{Y}, \bar{Z})$ be the corresponding optimal trajectory and $(p, q, k)$ be the solution to the adjoint equation 4.12). Then, for any $t \in\{0,1, \ldots, T\}$ and any $v \in U_{t}$, we have

$$
\left\langle H_{u}\left(t, \bar{u}_{t}, \bar{X}_{t}, \bar{Y}_{t}, \bar{Z}_{t}, p_{t}, q_{t}, k_{t}\right), v-\bar{u}_{t}\right\rangle \leq 0, P-a . s . .
$$


Proof. From the expression of $\xi_{t}, p_{t}$ for $t \in\{0,1, \ldots, T-1\}$, we have

$$
\begin{aligned}
\Delta\left\langle\xi_{t}, p_{t}\right\rangle= & \left\langle\xi_{t+1}, \Delta p_{t}\right\rangle+\left\langle\Delta \xi_{t}, p_{t}\right\rangle \\
= & \left\langle\xi_{t+1},-b_{x}^{*}(t+1) p_{t+1}-\sigma_{x}^{*}(t+1) q_{t+1}+f_{x}^{*}(t+1) k_{t+1}+l_{x}(t+1)\right\rangle \\
& +\left\langle\left[\sigma_{x}(t) \xi_{t}+\sigma_{y}(t) \eta_{t}+\sigma_{z}(t) \zeta_{t}+\delta_{t s} \varepsilon \sigma_{i u}(t) \Delta v\right] \Delta W_{t}, q_{t} \Delta W_{t}\right\rangle \\
& +\left\langle b_{x}(t) \xi_{t}+b_{y}(t) \eta_{t}+b_{z}(t) \zeta_{t}+\delta_{t s} b_{u}(t) \varepsilon \Delta v, p_{t}\right\rangle+\Phi_{t},
\end{aligned}
$$

where

$$
\begin{aligned}
\Phi_{t}= & \left\langle\xi_{t}+b_{x}(t) \xi_{t}+b_{y}(t) \eta_{t}+b_{z}(t) \zeta_{t}+\delta_{t s} b_{u}(t) \varepsilon \Delta v, q_{t} \Delta W_{t}\right\rangle \\
& +\left\langle\left[\sigma_{x}(t) \xi_{t}+\sigma_{y}(t) \eta_{t}+\sigma_{z}(t) \zeta_{t}+\delta_{t s} \varepsilon \sigma_{i u}(t) \Delta v\right] \Delta W_{t}, p_{t}\right\rangle \\
& +\left\langle\xi_{t}+b_{x}(t) \xi_{t}+b_{y}(t) \eta_{t}+b_{z}(t) \zeta_{t}+\delta_{t s} b_{u}(t) \varepsilon \Delta v, \Delta Q_{t}\right\rangle \\
& +\left\langle\left[\sigma_{x}(t) \xi_{t}+\sigma_{y}(t) \eta_{t}+\sigma_{z}(t) \zeta_{t}+\delta_{t s} \varepsilon \sigma_{i u}(t) \Delta v\right] \Delta W_{t}, \Delta Q_{t}\right\rangle
\end{aligned}
$$

Since $W$ and $Q$ are square integrable martingale processes and $Q$ is strongly orthogonal to $W$, we have $\mathbb{E}\left[\Phi_{t}\right]=0$. Similarly,

$$
\begin{aligned}
\Delta\left\langle\eta_{t}, k_{t}\right\rangle= & \left\langle\Delta \eta_{t}, k_{t+1}\right\rangle+\left\langle\eta_{t}, \Delta k_{t}\right\rangle \\
= & \left\langle-f_{x}(t+1) \xi_{t+1}-f_{y}(t+1) \eta_{t+1}-f_{z}(t+1) \zeta_{t+1}-\delta_{(t+1) s} f_{u}(t+1) \varepsilon \Delta v, k_{t+1}\right\rangle \\
& +\left\langle\zeta_{t} \Delta W_{t},\left[f_{z}^{*}(t) k_{t}-b_{z}^{*}(t) p_{t}-\sigma_{z}^{*}(t) q_{t}+l_{z}(t)\right] \Delta W_{t}\right\rangle \\
& +\left\langle\eta_{t}, f_{y}^{*}(t) k_{t}-b_{y}^{*}(t) p_{t}-\sigma_{y}^{*}(t) q_{t}+l_{y}(t)\right\rangle+\Psi_{t},
\end{aligned}
$$

where

$$
\begin{aligned}
\Psi_{t}= & \left\langle\zeta_{t} \Delta W_{t}, k_{t}+f_{y}^{*}(t) k_{t}-b_{y}^{*}(t) p_{t}-\sigma_{y}^{*}(t) q_{t}+l_{y}(t)\right\rangle \\
& +\left\langle\eta_{t},\left[f_{z}^{*}(t) k_{t}-b_{z}^{*}(t) p_{t}-\sigma_{z}^{*}(t) q_{t}+l_{z}(t)\right] \Delta W_{t}\right\rangle \\
& +\left\langle k_{t}+f_{y}^{*}(t) k_{t}-b_{y}^{*}(t) p_{t}-\sigma_{y}^{*}(t) q_{t}+l_{y}(t), \Delta V_{t}\right\rangle \\
& +\left\langle\left[f_{z}^{*}(t) k_{t}-b_{z}^{*}(t) p_{t}-\sigma_{z}^{*}(t) q_{t}+l_{z}(t)\right] \Delta W_{t}, \Delta V_{t}\right\rangle .
\end{aligned}
$$

Furthermore,

$$
\begin{aligned}
& \mathbb{E}\left\langle\left[\sigma_{x}(t) \xi_{t}+\sigma_{y}(t) \eta_{t}+\sigma_{z}(t) \zeta_{t}+\delta_{t s} \varepsilon \sigma_{i u}(t) \Delta v\right] \Delta W_{t}, q_{t} \Delta W_{t}\right\rangle \\
& =\mathbb{E}\left\langle\sigma_{x}(t) \xi_{t}+\sigma_{y}(t) \eta_{t}+\sigma_{z}(t) \zeta_{t}+\delta_{t s} \varepsilon \sigma_{i u}(t) \Delta v, q_{t} \mathbb{E}\left[\Delta W_{t}^{2} \mid \mathcal{F}_{t}\right]\right\rangle \\
& =\mathbb{E}\left[\sum_{i=1}^{d}\left\langle\xi_{t}, \sigma_{i x}^{*}(t) q_{t} e_{i}\right\rangle\right]
\end{aligned}
$$


and

$$
\begin{aligned}
& \mathbb{E}\left\langle\zeta_{t} \Delta W_{t},\left[f_{z}^{*}(t) k_{t}-b_{z}^{*}(t) p_{t}-\sigma_{z}^{*}(t) q_{t}+l_{z}(t)\right] \Delta W_{t}\right\rangle \\
& =\mathbb{E}\left\langle\zeta_{t},\left[f_{z}^{*}(t) k_{t}-b_{z}^{*}(t) p_{t}-\sigma_{z}^{*}(t) q_{t}+l_{z}(t)\right] \mathbb{E}\left[\Delta W_{t}^{2} \mid \mathcal{F}_{t}\right]\right\rangle \\
& =\mathbb{E}\left\langle\sum_{j=1}^{d} \zeta_{t} e_{j} \Delta W_{t}^{j}, \sum_{i=1}^{d} f_{z_{i}}^{*}(t) k_{t} \Delta W_{t}^{i}\right\rangle \\
& =\mathbb{E}\left[\sum_{i=1}^{d}\left\langle f_{z_{i}}(t) \zeta_{t} e_{i}, k_{t}\right\rangle\right] .
\end{aligned}
$$

Then, we obtain

$$
\begin{aligned}
& \mathbb{E}\left[\Delta\left(\left\langle\xi_{t}, p_{t}\right\rangle+\left\langle\eta_{t}, k_{t}\right\rangle\right)\right] \\
= & \mathbb{E}\left\langle-b_{x}(t+1) \xi_{t+1}, p_{t+1}\right\rangle+\left\langle b_{x}(t) \xi_{t}, p_{t}\right\rangle-\left\langle\xi_{t+1}, \sigma_{x}^{*}(t+1) q_{t+1}\right\rangle+\left\langle\xi_{t}, \sigma_{x}^{*}(t) q_{t}\right\rangle \\
& -\left\langle f_{y}(t+1) \eta_{t+1}, k_{t+1}\right\rangle+\left\langle f_{y}(t) \eta_{t}, k_{t}\right\rangle-\left\langle f_{z}(t+1) \zeta_{t+1}, k_{t+1}\right\rangle+\left\langle f_{z}(t) \zeta_{t}, k_{t}\right\rangle \\
& +\left\langle l_{x}(t+1), \xi_{t+1}\right\rangle+\left\langle l_{y}(t), \eta_{t}\right\rangle+\left\langle l_{z}(t), \zeta_{t}\right\rangle+\varepsilon\left\langle\delta_{t s} b_{u}(t) \Delta v, p_{t}\right\rangle+\delta_{t s} \varepsilon\left\langle\sigma_{u}(t) \Delta v, q_{t}\right\rangle \\
& -\varepsilon\left\langle\delta_{(t+1) s} f_{u}(t+1) \Delta v, k_{t+1}\right\rangle .
\end{aligned}
$$

Therefore,

$$
\begin{aligned}
& -\mathbb{E}\left\langle h_{x}\left(\bar{X}_{T}\right), \xi_{T}\right\rangle \\
= & \mathbb{E}\left[\left\langle\xi_{T}, p_{T}\right\rangle+\left\langle\eta_{T}, k_{T}\right\rangle-\left\langle\xi_{0}, p_{0}\right\rangle-\left\langle\eta_{0}, k_{0}\right\rangle\right] \\
= & \sum_{t=0}^{T-1} \mathbb{E} \Delta\left(\left\langle\xi_{t}, p_{t}\right\rangle+\left\langle\eta_{t}, k_{t}\right\rangle\right) \\
= & \mathbb{E}\left[\left\langle b_{x}(0) \xi_{0}, p_{0}\right\rangle+\sum_{i=1}^{d}\left\langle\xi_{0}, \sigma_{x}^{*}(0) q_{0}\right\rangle+\left\langle f_{y}(0) \eta_{0}, k_{0}\right\rangle+\left\langle f_{z}(0) \zeta_{0}, k_{0}\right\rangle\right] \\
& +\sum_{t=0}^{T-1} \mathbb{E}\left[\left\langle l_{x}(t), \xi_{t}\right\rangle+\left\langle l_{y}(t), \eta_{t}\right\rangle+\left\langle l_{z}(t), \zeta_{t}\right\rangle\right] \\
& +\sum_{t=0}^{T} \delta_{t s} \varepsilon \mathbb{E}\left[\left\langle b_{u}^{*}(t) p_{t}, \Delta v\right\rangle+\left\langle\sigma_{u}^{*}(t) q_{t}, \Delta v\right\rangle-\left\langle f_{u}^{*}(t) k_{t}, \Delta v\right\rangle\right] .
\end{aligned}
$$

Notice that $\xi_{0}=0, k_{0}=0$. So

$$
\begin{aligned}
& \mathbb{E} \sum_{t=0}^{T-1}\left[\left\langle l_{x}(t), \xi_{t}\right\rangle+\left\langle l_{y}(t), \eta_{t}\right\rangle+\left\langle l_{z}(t), \zeta_{t}\right\rangle\right]+\mathbb{E}\left\langle h_{x}\left(\bar{X}_{T}\right), \xi_{T}\right\rangle \\
= & -\varepsilon \mathbb{E}\left[\left\langle b_{u}^{*}(s) p_{s}+\sigma_{u}^{*}(s) q_{s}-f_{u}^{*}(s) k_{s}, \Delta v\right\rangle\right] .
\end{aligned}
$$

Since $\lim _{\varepsilon \rightarrow 0} \frac{1}{\varepsilon}\left[J\left(u^{\varepsilon}(\cdot)\right)-J(\bar{u}(\cdot))\right] \geq 0$, we obtain

$$
\mathbb{E}\left[\left\langle b_{u}^{*}(s) p_{s}+\sigma_{u}^{*}(s) q_{s}-f_{u}^{*}(s) k_{s}-l_{u}(s), \Delta v\right\rangle\right] \leq 0 .
$$

Then, (4.13) holds due to that $s$ is taking arbitrarily. This completes the proof. 


\section{References}

[1] Bender, C., \& Zhang, J. (2008). Time discretization and Markovian iteration for coupled FBSDEs. The Annals of Applied Probability, 18(1), 143-177.

[2] Bensoussan, A. (1982). Lectures on stochastic control. In Nonlinear filtering and stochastic control (pp. 1-62). Springer, Berlin, Heidelberg.

[3] Bielecki, T. R., Cialenco, I., \& Chen, T. (2015). Dynamic conic finance via backward stochastic difference equations. SIAM Journal on Financial Mathematics, 6(1), 1068-1122.

[4] Bismut, J. M. (1978). An introductory approach to duality in optimal stochastic control. SIAM review, 20(1), 62-78.

[5] Cohen, S. N., \& Elliott, R. J. (2010). A general theory of finite state backward stochastic difference equations. Stochastic Processes and their Applications, 120(4), 442-466.

[6] Cohen, S. N., \& Elliott, R. J. (2011). Backward stochastic difference equations and nearly time-consistent nonlinear expectations. SIAM Journal on Control and Optimization, 49(1), 125-139.

[7] Delarue, F., \& Menozzi, S. (2006). A forward-backward stochastic algorithm for quasi-linear PDEs. The Annals of Applied Probability, 16(1), 140-184.

[8] Dokuchaev, N., \& Zhou, X. Y. (1999). Stochastic controls with terminal contingent conditions. Journal of Mathematical Analysis and Applications, 238(1), 143-165.

[9] El Karoui, N., \& Huang, S. J. (1997). A general result of existence and uniqueness of backward stochastic differential equations. Pitman Research Notes in Mathematics Series, 27-38.

[10] Huang, J., \& Shi, J. (2012). Maximum principle for optimal control of fully coupled forward-backward stochastic differential delayed equations*. ESAIM: Control, optimisation and calculus of variations, 18(4), 1073-1096.

[11] Huang, J., Wang, G., \& Xiong, J. (2009). A maximum principle for partial information backward stochastic control problems with applications. SIAM journal on Control and Optimization, 48(4), 21062117 .

[12] Kushner, H. J. (1972). Necessary conditions for continuous parameter stochastic optimization problems. SIAM Journal on Control, 10(3), 550-565.

[13] Lim, A. E., \& Zhou, X. Y. (2001). Linear-quadratic control of backward stochastic differential equations. SIAM journal on control and optimization, 40(2), 450-474.

[14] Lin, X., \& Zhang, W. (2015). A maximum principle for optimal control of discrete-time stochastic systems with multiplicative noise. IEEE Transactions on Automatic Control, 60(4), 1121-1126.

[15] Ji, S. \& Liu, H. (2018). Fully coupled forward-backward stochastic difference equations, arXiv. 
[16] Peng, S. (1990). A general stochastic maximum principle for optimal control problems. SIAM Journal on control and optimization, 28(4), 966-979.

[17] Peng, S. (1993). Backward stochastic differential equations and applications to optimal control. Applied Mathematics and Optimization, 27(2), 125-144.

[18] Pardoux, E., \& Peng, S. (1990). Adapted solution of a backward stochastic differential equation. Systems \& Control Letters, 14(1), 55-61.

[19] Schroder, M., \& Skiadas, C. (1999). Optimal consumption and portfolio selection with stochastic differential utility. Journal of Economic Theory, 89(1), 68-126.

[20] Stadje, M. (2010). Extending dynamic convex risk measures from discrete time to continuous time: A convergence approach. Insurance: Mathematics and Economics, 47(3), 391-404.

[21] Wang, G., \& Wu, Z. (2009). The maximum principles for stochastic recursive optimal control problems under partial information. IEEE Transactions on Automatic control, 54(6), 1230-1242.

[22] Williams, N. (2009). On dynamic principal-agent problems in continuous time. University of Wisconsin, Madison.

[23] Wu, Z. (1998). Maximum principle for optimal control problem of fully coupled forward-backward stochastic systems. Systems Science and Mathematical sciences, 3, 249-259.

[24] Wu, Z. (2013). A general maximum principle for optimal control of forward-backward stochastic systems. Automatica, 49(5), 1473-1480.

[25] Xu, W. (1995). Stochastic maximum principle for optimal control problem of forward and backward system. The ANZIAM Journal, 37(2), 172-185.

[26] Yong, J. (2010). Optimality variational principle for controlled forward-backward stochastic differential equations with mixed initial-terminal conditions. SIAM Journal on Control and Optimization, 48(6), 4119-4156.

[27] Xu, J., Zhang, H., \& Xie, L. (2017). Solvability of general linear forward and backward stochastic difference equations. Control Conference (pp.1888-1891). IEEE.

[28] Xu, J., Zhang, H., \& Xie, L. (2018). General linear forward and backward stochastic difference equations with applications. Automatica, 96, 40-50. 